\title{
The Impact of Frailty on Spine Surgery: Systematic Review on 10 years Clinical Studies
}

\author{
Francesca Veronesi ${ }^{1}$, Veronica Borsari ${ }^{1{ }^{*},}$, Lucia Martini ${ }^{1}$, Andrea Visani ${ }^{1}$, Alessandro \\ Gasbarrini $^{2}$, Giovanni Barbanti Brodano², Milena Fini ${ }^{1}$ \\ ${ }^{1}$ Complex Structure of Surgical Sciences and Technologies, IRCCS Istituto Ortopedico Rizzoli, Bologna, Italy. \\ ${ }^{2}$ Spine Surgery prevalently Oncologic and Degenerative, IRCCS Istituto Ortopedico Rizzoli, Bologna, Italy.
}

[Received July 24, 2020; Revised September 2, 2020; Accepted September 4, 2020]

\begin{abstract}
Frailty is a condition characterized by a high vulnerability to low-power stressor. Frailty increases with age and is associated with higher complications and mortality. Several indexes have been used to quantify frailty. Spine diseases, both degenerative and oncologic, frequently require surgery which is related to complications and mortality. Aim of the present systematic review was to collect the most frequently used frailty indexes in clinics to predict surgical outcomes in patients affected by spine diseases, taking into account gender differences. Three databases were employed, and 29 retrospective clinical studies were included in this systematic review. The identified spine pathologies were primary and metastatic spine tumors, adult spine deformity (ASD), degenerative spine disease (DSD), cervical deformity (CD) and other pathologies that affected lumbar spine or multiple spine levels. Eleven indexes were identified: modified Frailty Index (mFI), Adult spinal deformity frailty index (ASD-FI), mFI-5, Metastatic Spinal Tumor Frailty Index (MSTFI), Fried criteria, Cervical deformity frailty index (CD-FI), Spinal tumor frailty index (STFI), Frailty Phenotype criteria (FP), Frailty Index (FI), FRAIL scale and Modified CD-FI (mCD-FI). All these indexes correlated well with minor and major postoperative complications, mortality and length of stay in hospital. Results on gender differences and frailty are still conflicting, although few studies show that women are more likely to develop frailty and more complications in the post-operative period than men. This systematic review could help the surgeon in the adoption of frailty indexes, before the operation, and in preventing complications in frail patients.
\end{abstract}

Key words: frailty, spine diseases, frailty indexes, gender

Even if frailty condition has been known for more than 30 years, the definition of the frail phenotype was first given in geriatric literature by Fried in 2001 [1] and has gained wide attention only in the last years. Frailty is a biologic syndrome characterized by a high vulnerability to lowpower stressors, manifested clinically by decreased functional reserve and resilience, together with multiorgan dysfunction or multimorbidity [1]. A consensus conference in December of 2012, led by the International Association of Gerontology and Geriatrics and the World Health Organization, defined frailty as "a medical syndrome with multiple causes and contributors that is characterized by diminished strength, endurance, and reduced physiologic function that increases an individual's vulnerability for developing increased dependency and/or death' [2].

Several procedures have been proposed for the assessment of frailty, which rely on the measure of physical functions, as accumulation of deficits and frailty phenotype, which application depends on availability in the clinical setting, and/or self-reported items on strength, energy and weight loss [3, 4]. Moreover, specific tools have been developed in definite settings [5], thus

*Correspondence should be addressed to: Dr. Veronica Borsari, Complex Structure of Surgical Sciences and Technologies, IRCCS Istituto Ortopedico Rizzoli, 40136 Bologna, Italy. Email: veronica.borsari@ior.it.

Copyright: () 2020 Veronesi F. et al. This is an open-access article distributed under the terms of the Creative Commons Attribution License, which permits unrestricted use, distribution, and reproduction in any medium, provided the original author and source are credited. 
consensus on distinctive diagnostic criteria is still missing.

It is reported that the prevalence of frailty increases with age, from $4 \%$ for ages between 65 and 69 years to $26 \%$ for older than $85[6]$ and it is more frequent in females than in males $[6,7]$. The worldwide occurrence of frailty varies extensively between $4 \%$ and $59 \%$ due to the heterogeneity of study populations and the use of different screening tools that consider different criteria $[8$, 9].

The dramatic increase in old-aged population is one of the main concerns. According to the United Nations, the proportion of global population over 65 years of age is expected to rise from $9 \%$ in 2019 to $16 \%$ by 2050 [10]. With increase in life expectancy, chronic noncommunicable diseases have become prevalent together with a rising number of elderly patients affected by degenerative, traumatic, oncologic or infective pathologies.

These demographic and epidemiologic transitions have a deep impact on health care provision and economic burden. A recent study from Norway highlights that patients over 65 years represent only $15 \%$ of the population, but are responsible for almost half of the total healthcare cost [11]. Moreover, in a prospective cohort study from US, pre-frailty and frailty are associated with higher subsequent total healthcare costs in older community-dwelling men [12].

Thus, the preservation of independence in aged people and the prevention of disability are priority major challenges and frailty is becoming an increasingly important concept both for its deep impact on health outcomes and impaired quality of life.

Frailty is associated with increasing disability, hospitalization, adverse health outcomes and death [1]. A number of observational studies have also shown that frailty worsened postoperative outcomes as morbidity, mortality and length of stay (LOS) [13-15] and the severity of frailty syndrome has been reported to be directly correlated with post-surgical mortality rates and complications [16].

As frailty is correlated to general surgery outcome, it might also predict the outcome in patients undergoing spine surgery, who have been reported to record a high rate of intra- and post-operative complications [17-20]. Degenerative disorders of the spine are very frequent, with $90 \%$ of adults showing some degree of degeneration of the lumbar disk or spine by age 50 [21]. Degeneration of the spine includes a wide variety of clinical conditions, as disk degeneration, spinal stenosis, facet hypertrophy, osteophytosis, foraminal stenosis and instability, leading to back pain and/or associated neurological signs [21]. Back pain affects 15\%-20\% of adults each year [22] and about 17.000 new cases of spinal column injuries are reported every year in US [23]. In addition, vertebral fractures are the most frequent osteoporotic fractures among aged people, together with proximal femoral and wrist fractures [24]. Spinal metastases affect $30-70 \%$ of patients with primary tumors and can lead to spinal cord compression, pain, spinal instability and pathologic fractures [25].

Hypothesis of the present study is that frailty may play a key role in the outcome of spinal surgery and may provide a useful tool for risk prediction, facilitating the decision-making process and surgery planning in patients affected by spine disease. To the best of our knowledge, only one systematic review, on associations between frailty and spine disease, has yet been conducted [26].

The aim of this study was to systematically review 10-year clinical data regarding association between frailty and outcomes after surgery for spine disease, by identifying the most used frailty indices in spine surgery.

\section{MATERIALS AND METHODS}

Three databases were employed to individuate clinical studies included in the present systematic review: www.pubmed.com, www.webofknowledge.com and www.scopus.com (Fig. 1).

In the Pubmed database the search was performed with the following meshes: ((("Spine"[Mesh] OR "Osteoarthritis, Spine"[Mesh] OR "Rigid spine syndrome" [Supplementary Concept] OR "Spinal Dysraphism"[Mesh] OR "Spinal Osteochondrosis"[Mesh] OR "Scheuermann Disease"[Mesh] OR "Dendritic Spines"[Mesh] OR "Camptocormia" [Supplementary Concept] OR "Microcephaly cervical spine fusion anomalies" [Supplementary Concept] OR "Ossification of the posterior longitudinal ligament of the spine" [Supplementary Concept])) OR ("Spinal Diseases"[Mesh] AND) AND (Frailty))). The limits were English language and publication date 2010/01/01-2019/31/12. With this search strategy, 68 studies were found.

In the Web of knowledge database, the search was performed with "(spine disease) AND (frailty)" keywords and the limits were English language, article document type and timespan 2010-2019, founding 25 studies.

In the Scopus database, "(spine disease) AND (frailty)" were also employed as keywords with the following limits: English language, article type and 20102019 years of publication and 70 studies were obtained. 


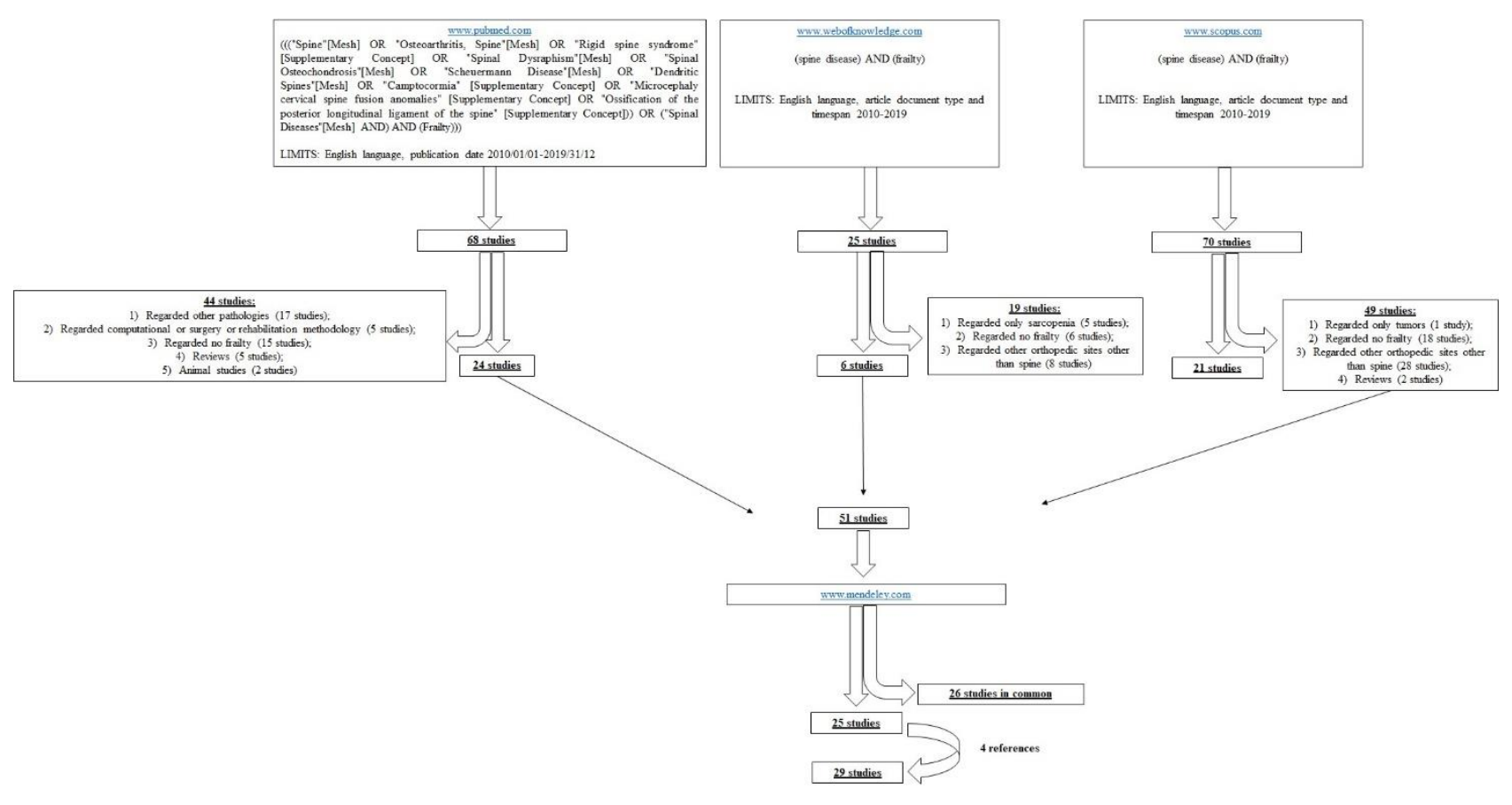

Figure 1. Schematic representation of the search strategy.

Relevant studies were firstly screened through title and abstract by one author (FV) and the studies that did not match the argument of the review were excluded. In the first database, 24 studies were included and 44 In the first database, 24 studies were included and 44 excluded because not inherent: they regarded other pathologies (17 studies), computational or surgery or rehabilitation methodology (5 studies), not regarded frailty (15 studies) or they were reviews (5 studies) and animal studies (2 studies). In the second database, 19 studies were excluded because regarded: 1) only sarcopenia (5 studies), no frailty (6 studies) or other orthopedic sites other than spine (8 studies). In the third database, 49 studies were excluded because they regarded only tumors (1 study), no frailty (18 studies), other orthopedic sites other than spine (28 studies) or they were reviews (2 studies).

Fifty-one studies were accepted and then submitted to www.mendeley.com to eliminate duplicates, finding 26 studies in common.

The full text of the remaining 25 studies were examined by two authors (FV, VB) and the studies characteristics and results were summarized in Table 1. Finally, an additional search was performed by reading the reference lists of the 25 studies, founding further 4 studies.

So, a total of 29 clinical studies were included in the present systematic review (Fig. 1).

\section{Assessment of Methodological Quality}

Two authors (FV, VB) independently assessed the methodological quality of the included studies with Quality in Prognosis Studies (QUIPS) tool [27] and summarized in Table 2. More precisely the tool assessed, for each study, 6 domains: 1) study participation, 2) study attrition, 3) prognostic factor measurement, 4) outcome measurement, 5) study confounding, and 6) statistical analysis and reporting. In case of disagreement, the two authors found an agreement by discussing their evaluations.

\section{RESULTS}

As observed in Table 1, the 29 clinical studies included in this systematic review, can be divided into two groups of spinal diseases, 5 dealing with spinal tumors (primary or metastases) [28-32] and 24 dealing with other spine diseases, as adult spine deformity (ASD) [33-39], degenerative spine disease (DSD) [40-42], cervical deformity (CD) [43-45], lumbar spine diseases [46-50], diseases at different spine levels [51-55] or vertebral fractures [56]. All were retrospective clinical studies and used several different databases, with different years of recruitment, to enroll patients: Nationwide Inpatient Sample (NIS) database in 2002-2011 years [28, 29], American College of Surgeons National Surgical Quality Improvement Program (ACS-NSQIP) database in 20082014 [32], 2006-2012 [39, 41], 2011-2014 [46], 20102014 [47], 2005-2012 [48], 2012-2016 [49], 2007-2012 
[51], 2006-2010 [52] or 2006-2015 [53] years, a multicenter, prospective database maintained by the International Spine Study Group (ISSG) or European Spine Study Group (ESSG) database in 2010-2014 years [34-36, 43], Spine Surgery Database of Adverse Events in 2009-2013 years [40], a multicenter database of 13 spine surgery centers across the USA in 2013-2018 years [44,
45], Spinal center of a tertiary-care teaching hospital database in 2014-2017 years [50, 56], Mount Sinai Electronic Scheduling system in 2013-2014 years [54] and not defined hospital database in which the research was carried in 2010-2015 [30], 2009-2016 [31], 20052015 [42], 2010-2013 [55], not specified [33, 37, 38] years.

Table 1. Outcomes of the 29 clinical studies performed in frail patients affected by spine pathologies.

\begin{tabular}{|c|c|c|}
\hline Aim & $\begin{array}{l}\text { Database } \\
\text { employed }\end{array}$ & Spine pathology \\
\hline $\begin{array}{l}\text { Development } \\
\text { of STFI to } \\
\text { predict p.o } \\
\text { complications, } \\
\text { LOS, in- } \\
\text { hospital } \\
\text { mortality }\end{array}$ & $\begin{array}{l}\text { NIS } \\
\text { database } \\
(2002- \\
2011)\end{array}$ & $\begin{array}{l}\text { Surgery for benign } \\
\text { or malignant } \\
\text { primary spinal } \\
\text { neoplasms in } \\
\text { vertebral column, } \\
\text { sacrum and coccyx }\end{array}$ \\
\hline $\begin{array}{l}\text { Development } \\
\text { of MSTFI to } \\
\text { predict } \\
\text { perioperative } \\
\text { complications, } \\
\text { in-hospital } \\
\text { mortality, } \\
\text { LOS }\end{array}$ & $\begin{array}{l}\text { NIS } \\
\text { database } \\
(2002- \\
2011)\end{array}$ & $\begin{array}{l}\text { Surgery for spinal } \\
\text { metastases with a } \\
\text { primary tumor in } \\
\text { breast, lung, } \\
\text { thyroid, kidney or } \\
\text { prostate }\end{array}$ \\
\hline
\end{tabular}

Evaluation of mFI to predict mortality

\begin{tabular}{|c|c|}
\hline $\begin{array}{l}\text { Evaluation of } \\
\text { FI to predict } \\
\text { mortality or } \\
\text { complications }\end{array}$ & $\begin{array}{l}\text { A } \\
\text { quaternal } \\
\text { y referral } \\
\text { center } \\
\text { database } \\
(2009- \\
2016)\end{array}$ \\
\hline $\begin{array}{l}\text { Evaluation of } \\
\text { mFI to predict } \\
\text { mortality, } \\
\text { major and } \\
\text { minor } \\
\text { complications, } \\
\text { LOS }\end{array}$ & $\begin{array}{l}\text { ACS- } \\
\text { NSQIP } \\
\text { database } \\
(2008- \\
2014)\end{array}$ \\
\hline
\end{tabular}

A hospital
coding
database
(2010-
2015)

Surgery for spinal metastasis with primary tumors in prostate, unknown sites, breast, lungs, bladder, kidney, cervix, thyroid.

Metastasis located in Cervical,

Thoracic, Lumbar, Cervicothoracic/Thoracolumbar junctions, Sacrum locations Spinal metastases with primary tumor located in breast, lung, kidney

Surgery for
primary and
metastatic tumors
in extradural,
intradural
extramedullary and
intramedullary
locations

\begin{tabular}{|c|c|c|}
\hline $\begin{array}{l}\text { Pts } \\
\text { characteristics }\end{array}$ & $\begin{array}{l}\text { Frailty } \\
\text { evaluation }\end{array}$ & Results \\
\hline $\begin{array}{l}1589 \text { pts }(28-61 \\
\text { yrs). } \\
823 \text { men, } 766 \\
\text { women }\end{array}$ & $\begin{array}{l}\text { STFI } \\
\text { NF }=71.7 \% \\
\text { Mild frailty }= \\
20.1 \% \\
\text { Moderate } \\
\text { frailty }=6.0 \% \\
\text { SF }=2.2 \%\end{array}$ & $\begin{array}{l}\text { Mild frailty, } \\
\text { moderate frailty } \\
\text { and SF: } \uparrow \text { all } \\
\text { complications and } \\
\text { LOS than NF }\end{array}$ \\
\hline $\begin{array}{l}4583 \text { pts }(54-70 \\
\text { yrs). } \\
2650 \text { men, } 1931 \\
\text { women }\end{array}$ & $\begin{array}{l}\text { MSTFI } \\
\text { NF = } 17.2 \% ; \\
\text { Mild Frailty = } \\
40.1 \% ; \\
\text { Moderate } \\
\text { Frailty = } \\
24.7 \% ; \\
\text { SF }=18.0 \%\end{array}$ & $\begin{array}{l}\text { Moderate frailty } \\
\text { and SF: } \uparrow \\
\text { mortality than NF. } \\
\text { Mild frailty, } \\
\text { moderate frailty } \\
\text { and SF: } \uparrow \text { major } \\
\text { complications and } \\
\text { LOS than NF }\end{array}$ \\
\hline
\end{tabular}

\author{
Outcomes \\ P.o. complications \\ (acute respiratory \\ distress syndrome, \\ pleurisy, \\ pneumothorax, \\ pulmonary collapse, \\ reintubation, \\ pneumonia, PE, \\ cardiac arrest, MI, \\ iatrogenic stroke, acute \\ renal failure); \\ mortality; LOS \\ Perioperative \\ complications \\ (unplanned \\ reintubation, cardiac \\ arrest, pneumonia, MI, \\ $\mathrm{PE}$, sepsis, acute renal \\ failure, shock, \\ pleurisy/pneumothorax \\ /pulmonary collapse, \\ adult respiratory \\ distress syndrome, \\ iatrogenic stroke); \\ Mortality; LOS \\ Ref

41 pts $(64 \pm 9.1 \quad \mathrm{mFI} \quad \mathrm{mFI}$ poorly $\quad$ Mortality

correlated with

survival

26 men, 15 women

\section{8 pts (35-84 mFI;}

yrs).

57 men, 51

women

\section{MSTFI}

mFI: correlated

with

complications.

MSTFI: correlated

with mortality

\section{0 pts}

(57 \pm 16 yrs).

1172 men, 998

women

$\mathrm{mFI}$

$\mathrm{F}: \uparrow$ mortality and

LOS than NF

\author{
Mortality; major \\ postoperative \\ complications \\ (prolonged intubation \\ of $48 \mathrm{hrs}$ or more, \\ return to the operating \\ room, unplanned re- \\ intubation, sepsis, \\ venous \\ thromboembolism,
}




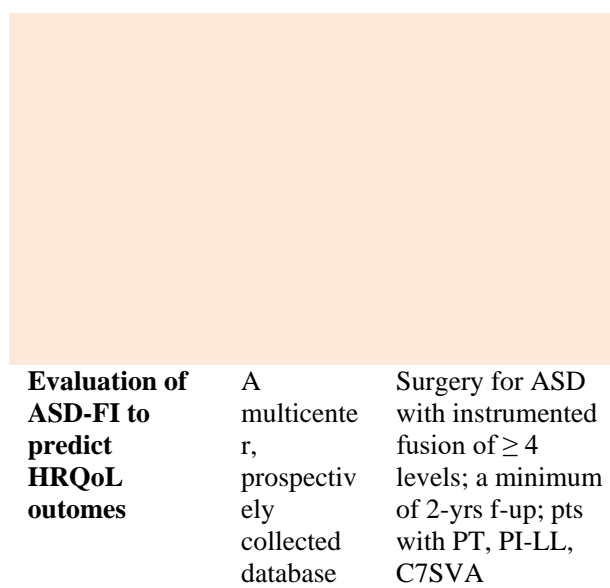

\begin{tabular}{|c|c|c|}
\hline $\begin{array}{l}\text { Development } \\
\text { and evaluation } \\
\text { of ASD-FI to } \\
\text { predict } \\
\text { complications, } \\
\text { LOS, } \\
\text { reoperation } \\
\text { rate }\end{array}$ & $\begin{array}{l}\text { Multicent } \\
\text { er, } \\
\text { prospectiv } \\
\text { e database } \\
\text { maintaine } \\
\mathrm{d} \text { by the } \\
\text { ISSG } \\
\text { (2010- }\end{array}$ & $\begin{array}{l}\text { Surgery for ASD } \\
\text { with scoliosis } \\
\text { (major curve } \geq \\
20^{\circ} \text { ), thoracic } \\
\text { kyphosis } \geq 60^{\circ}, \mathrm{PT} \\
\geq 20^{\circ}, \mathrm{C} 7 \mathrm{SVA}>5 \\
\mathrm{~cm} ; \text { minimum of } 2 \\
\text { yrs of f-up }\end{array}$ \\
\hline
\end{tabular}

Validation

and evaluation

of ASD-FI to

predict

complications,

reoperation

rate, LOS

\begin{tabular}{|c|c|}
\hline $\begin{array}{l}\text { Multicent } \\
\text { er } \\
\text { database } \\
\text { maintaine } \\
\text { d by } \\
\text { ESSG } \\
(2010- \\
2014)\end{array}$ & $\begin{array}{l}\text { Surgery for ASD } \\
\text { with scoliosis } \\
\text { (major curve } \geq \\
20^{\circ} \text { ), thoracic } \\
\text { kyphosis } \geq 60^{\circ}, \mathrm{PT} \\
\geq 20^{\circ}, \mathrm{C} 7 \mathrm{SVA}>5 \\
\text { cm; age } \geq 18 \text { yrs; } \\
\text { minimum of } 2 \text { yrs } \\
\text { of f-up }\end{array}$ \\
\hline
\end{tabular}

\begin{tabular}{|c|c|c|}
\hline $\begin{array}{l}\text { Validation } \\
\text { and evaluation } \\
\text { of ASD-FI to } \\
\text { predict major } \\
\text { complications, } \\
\text { LOS }\end{array}$ & $\begin{array}{l}\text { Multicent } \\
\text { er } \\
\text { database } \\
\text { maintaine } \\
\text { d by } \\
\text { ESSG }\end{array}$ & $\begin{array}{l}\text { Surgery for ASD } \\
\text { with scoliosis } \\
\text { (major curve } \\
\geq 20^{\circ} \text { ), thoracic } \\
\text { kyphosis } \geq 60^{\circ}, \mathrm{PT} \\
\geq 20^{\circ} \text {, or C7SVA } \\
>5 \mathrm{~cm}\end{array}$ \\
\hline
\end{tabular}

$\begin{array}{lll}417 \text { pts } & \text { ASD-FI } & \text { F and SF: } \uparrow \text { major } \\ (57.67 \pm 1.13 & \mathrm{NF}=41 \% ; & \text { intraoperative and } \\ \text { yrs). } & \mathrm{F}=39 \% ; & \text { p.o. complications, } \\ 82 \text { men, } 335 & \mathrm{SF}=20 \% & \text { any complications, } \\ \text { women } & & \text { LOS, junctional } \\ & & \text { kyphosis than NF } \\ & \text { SF: } \uparrow \text { reoperation, } \\ & & \text { PJK, wound } \\ & \text { dehiscence, deep } \\ & & \text { wound infection } \\ & & \\ & & \end{array}$

$\begin{array}{lll}266 \mathrm{pts} & \text { ASD-FI } & \text { SF: } \uparrow \text { major } \\ (54 \pm 2.03 \mathrm{yrs}) . & \mathrm{NF}=51 \% ; & \text { intraoperative or } \\ 63 \text { men, 203 } & \mathrm{F}=34 \% ; & \text { p.o. complications, } \\ \text { women } & \mathrm{SF}=15 \% & \text { PJK, wound }\end{array}$

infection,

reoperation, LOS

than NF.

$\mathrm{F}: \uparrow$ major

complications

LOS than NF

coma, stroke, cardiac arrest, septic shock, MI, surgical site/organ space infection, acute renal failure); minor complications (perioperative blood transfusion, UTI, pneumonia, renal insufficiency, wound dehiscence); LOS SCB and change in 33 ODI, SF-36 PCS, back pain and leg pain scores

Major complications 34 (intraoperative vascular, visceral, or neurological injury, postoperative deep infection, PE, junctional failure, similar complications); Deep wound infection rate; Wound dehiscence incidence; LOS; PJK incidence; Pseudarthrosis incidence; Reoperation rate

Major perioperative 35 complications (intraoperative vascular, visceral, or neurologic injury, deep wound infection, PE, junctional failure, other similar complications); LOS; Reoperation; PJK incidence; Deep wound infection rate; Surgical complications (intraoperative and immediate p.o. complications);

Medical complications (stroke, DVT, PE, pneumonia, UTI) Major complications 36 yrs). 88 men, 179 women $\mathrm{NF}=39.33 \%$ $\mathrm{F}=38.58 \%$; $\mathrm{SF}=22.10 \%$
F: $\uparrow$ all complications than NF.

SF: $\uparrow$ minor, major or all complication, LOS than NF (intraoperative vascular, visceral, or neurologic injury, postoperative deep wound infection, PE, junctional failure, other similar complications); LOS; Overall complication incidence 


$\begin{array}{ll}\begin{array}{l}\text { Evaluation of } \\ \text { the treatment }\end{array} & \begin{array}{l}\text { Multicent } \\ \text { er }\end{array} \\ \text { status for } & \text { database } \\ \text { Frailty to } & \text { of one } \\ \text { predict } & \text { institute } \\ \text { complications } & \end{array}$

$\begin{array}{ll}\text { Evaluation of } & \text { Multicent } \\ \text { mFI-5 and } & \text { er } \\ \text { mFI-11 to } & \text { database } \\ \text { predict severe } & \text { of one } \\ \text { complications } & \text { institute }\end{array}$

complications
Surgery for ASD

with scoliosis

(major curve $\geq$

$\mathrm{cm}, \mathrm{PT} \geq 25^{\circ} ; 21$

yrs; $\geq 5$ fused

vertebral levels,

segmental pedicle

screw fixation

from the upper-

instrumented

vertebra to the

lower instrumented

vertebra; minimum of 2-yrs of f-up

Surgery for ASD

with scoliosis

(major curve $\geq$

$\left.30^{\circ}\right), \mathrm{C} 7 \mathrm{SVA} \geq 5$

$\mathrm{cm}, \mathrm{PT} \geq 25^{\circ} ; \geq 21$

yrs; $\geq 5$ fused

vertebral levels,

segmental pedicle

screw fixation

from the upper-

instrumented

vertebral to the

lower-instrumented

vertebral level;

minimum of 2-yrs

of f-up $\left.20^{\circ}\right), \mathrm{C} 7 \mathrm{SVA} \geq 5$

$\begin{array}{lll}240 \mathrm{pts} & \mathrm{mFI} & \mathrm{R}: \downarrow \text { perioperative } \\ (58.4 \pm 16.7 \mathrm{yrs}) . & \mathrm{NF}=59 \% ; & \text { complications, } 2 \\ 19 \text { men, 221 } & \mathrm{PF}=34 \% ; & \text { yrs overall } \\ \text { women } & \mathrm{F}=7 \% ; & \text { complications, p.o. } \\ & \mathrm{G}=72 \% ; & \text { C7SVA; } \uparrow \\ & \mathrm{PC}=28 \% & \text { incidence of } \mathrm{C}- \\ & & \text { D2, C-D3 } \\ & & \text { complication, } \\ & & \text { SRS22 function } \\ & & \text { than G and } \mathrm{PC}\end{array}$

Intraoperative and p.o.

37

complication

(surgical

complications,

surgical-site infection,

other infection,

excessive bleeding,

delirium,

cardiopulmonary,

gastrointestinal, or

renal diseases)

$\begin{array}{ll}281 \mathrm{pts} & \mathrm{mFI}-5 \\ (54.4 \pm 18.7 \mathrm{yrs}) & \mathrm{mFI} \\ & \mathrm{NF}=66 \% ; \\ & \mathrm{PF}=22 \% ; \\ & \\ & \\ & \\ & \\ & \\ & \\ 1001 \mathrm{pts} & \mathrm{mFI} \\ \text { (59 } 14 \text { yrs). } & \mathrm{NF}=38.86 \% ; \\ 460 \text { men, 541 } & \mathrm{PF}=58.14 \% ; \\ \text { women } & \mathrm{F}=3 \%\end{array}$

$\mathrm{mFI}-5$ and $\mathrm{mFI}$ :

excellent

concordance

across ASD

surgery.

mFI F: $\uparrow$ total

complications,

perioperative

complications,

implant-related

complications,

severe

complications.

mFI-5 F: $\uparrow$ severe

complications.

$\mathrm{mFI}-5$ and $\mathrm{mFI}-$

11: strong

predictive ability

for severe

complications

Surgery for ASD $\quad 1001 \mathrm{pts} \quad \mathrm{mFI} \quad \mathrm{F}: \uparrow$ mortality,

with spinal fusion

F: $\uparrow$ mortality,

for deformity; long

spinal fusion

P.o. complications

(pneumonia, sepsis,

DVT, PE,

woundcomplication,
Evaluation of mFI to predict

p.o.

complications,

mortality

\section{ACS-}

NSQIP

database

(2005-

2012)
PE/DVT, any p.o.

complications,

reoperation than $\mathrm{NF}$ deep infection, CNS complication,

sepsis/septic shock, cardiac arrest, acute renal failure, UTI, reoperation); Mortality occurring within 30 days

\begin{tabular}{|c|c|c|c|c|c|}
\hline $\begin{array}{l}\text { Evaluation of } \\
\text { mFI to predict } \\
\text { p.o. } \\
\text { complications, } \\
\text { LOS, } \\
\text { discharge to a } \\
\text { facility, in- } \\
\text { hospital } \\
\text { mortality }\end{array}$ & $\begin{array}{l}\text { Spine } \\
\text { Surgery } \\
\text { Database } \\
\text { of } \\
\text { Adverse } \\
\text { Events } \\
(2009- \\
2013)\end{array}$ & $\begin{array}{l}\text { Primary DSD with } \\
\text { spondylolisthesis, } \\
\text { lumbar stenosis } \\
\text { and disc herniation } \\
\text { at thoracolumbar } \\
\text { spine (T9-S1) } \\
\text { level }\end{array}$ & $\begin{array}{l}102 \text { pts }(68-78 \\
\text { yrs). } \\
51 \text { men, } 51 \\
\text { women }\end{array}$ & $\begin{array}{l}\mathrm{mFI} \\
\mathrm{NF}=59.8 \% \\
\mathrm{PF}=20.6 \% \\
\mathrm{~F}=19.6 \%\end{array}$ & $\begin{array}{l}\mathrm{mFI} \text { not associated } \\
\text { with incidence of } \\
\text { p.o. complications. } \\
\mathrm{F}: \uparrow \text { risk of } \\
\text { mortality than NF }\end{array}$ \\
\hline $\begin{array}{l}\text { Evaluation of } \\
\text { mFI to predict } \\
\text { p.o. } \\
\text { complications, } \\
\text { LOS, } \\
\text { discharge } \\
\text { disposition, } \\
\text { mortality }\end{array}$ & $\begin{array}{l}\text { ACS- } \\
\text { NSQIP } \\
\text { database } \\
(2006- \\
2012)\end{array}$ & $\begin{array}{l}\text { Elective or } \\
\text { semielective } \\
\text { surgery for DSD } \\
\text { with procedural } \\
\text { related to the spine }\end{array}$ & $\begin{array}{l}52,671 \mathrm{pts} \\
(56.1 \pm 14.5 \mathrm{yrs}) . \\
27389 \text { men, } \\
25282 \text { women }\end{array}$ & $\begin{array}{l}\mathrm{mFI} \\
\mathrm{NF}=46 \% \\
\mathrm{PF}=50 \% \\
\mathrm{~F}=4 \%\end{array}$ & $\begin{array}{l}\text { F: } \uparrow \text { major } \\
\text { complication, } \\
\text { reoperation for } \\
\text { p.o. infection, } \\
\text { LOS, discharge to } \\
\text { a new facility, 30- } \\
\text { day mortality than } \\
\text { NF }\end{array}$ \\
\hline $\begin{array}{l}\text { Evaluation of } \\
\text { the } \\
\text { relationship } \\
\text { between mFI } \\
\text { and BMI }\end{array}$ & $\begin{array}{l}\text { Not } \\
\text { specified } \\
(2005- \\
2015)\end{array}$ & $\begin{array}{l}\text { Surgery for DSD at } \\
\text { cervical, thoracic } \\
\text { and lumbar levels }\end{array}$ & $\begin{array}{l}1970 \text { pts } \\
(58.1 \pm 5.91 \text { yrs }) . \\
1045 \text { men, } \\
925 \text { women }\end{array}$ & $\begin{array}{l}\mathrm{mFI} \\
\mathrm{NF}=42.39 \% \\
\mathrm{PF}=54.57 \% ; \\
\mathrm{F}=3.05 \% \\
\mathrm{BMI} \\
\text { underweight }= \\
<18.5 ;\end{array}$ & $\begin{array}{l}\text { mFI: positive } \\
\text { correlation with } \\
\text { complications and } \\
\text { negative } \\
\text { correlation with } \\
\text { BMI. }\end{array}$ \\
\hline
\end{tabular}

Any perioperative AEs $\quad 40$ (intraoperative and p.o. complications); LOS; p.o. discharge to a facility; In-hospital mortality 


\begin{tabular}{|c|c|c|c|c|c|c|c|}
\hline & & & & $\begin{array}{l}\text { normal weight } \\
=18.5-25 ; \\
\text { overweight }= \\
25.0-29.9 ; \\
\text { Obese }=>30.0\end{array}$ & $\begin{array}{l}\text { Underweight: } \uparrow \\
\text { prefrailty and } \\
\text { frailty. } \\
\text { Obese: } \uparrow \text { frailty. } \\
\text { Underweight, } \\
\text { Obese, } \mathrm{PF} \text { and F: } \uparrow \\
\text { p.o. complications. } \\
\text { Underweight/norm } \\
\text { al weight+PF/F, } \\
\text { overweight+F and } \\
\text { obese+NF/F: } \uparrow \\
\text { p.o. complications }\end{array}$ & $\begin{array}{l}\text { parenteral nutrition, } \\
\text { requiring radiological, } \\
\text { endoscopic, or surgical } \\
\text { interventions, life- } \\
\text { threatening } \\
\text { complications } \\
\text { requiring ICU } \\
\text { management, death) }\end{array}$ & \\
\hline $\begin{array}{l}\text { Evaluation of } \\
\text { CD-FI to } \\
\text { predict } \\
\text { preoperative } \\
\text { risk, } \\
\text { complications, } \\
\text { LOS, } \\
\text { discharge } \\
\text { disposition }\end{array}$ & $\begin{array}{l}\text { Multicent } \\
\text { er, } \\
\text { prospectiv } \\
\text { e database } \\
\text { maintaine } \\
\text { d by ISSG } \\
(2009- \\
2015)\end{array}$ & $\begin{array}{l}\text { Surgery for CD: } \\
\text { Cervical scoliosis } \\
\text { (major angle } \geq 10^{\circ} \text { ) } \\
\text { and cervical } \\
\text { kyphosis (major } \\
\text { angle }>10^{\circ} \text { ); } \\
\text { minimum 1-yr f-up }\end{array}$ & $\begin{array}{l}61 \text { pts }(61 \pm 2.7 \\
\text { yrs). } \\
24 \text { men, } 37 \\
\text { women }\end{array}$ & $\begin{array}{l}\text { CD-FI. } \\
N F=27.9 \% \\
F=55.7 \% \\
S F=16.4 \%\end{array}$ & $\begin{array}{l}\text { SF: } \uparrow \text { major } \\
\text { complications, } \\
\text { medical } \\
\text { complications than } \\
\text { NF }\end{array}$ & $\begin{array}{l}\text { Major complications } \\
\text { (intraoperative } \\
\text { vascular, visceral, or } \\
\text { neurologic injury, } \\
\text { postoperative deep } \\
\text { infection, PE, } \\
\text { junctional failure); } \\
\text { LOS; Discharge } \\
\text { disposition; } \\
\text { Medical/surgical } \\
\text { complications (most } \\
\text { intraoperative } \\
\text { complications and } \\
\text { immediate } \\
\text { postoperative } \\
\text { complications related } \\
\text { to surgical } \\
\text { technique/error, stroke, } \\
\text { DVT, PE, pneumonia, } \\
\text { UTI) }\end{array}$ & 43 \\
\hline $\begin{array}{l}\text { Evaluation of } \\
\text { mCD-FI to } \\
\text { predict p.o. } \\
\text { clinical } \\
\text { outcomes, } \\
\text { complications, } \\
\text { HRQoL, } \\
\text { mortality }\end{array}$ & $\begin{array}{l}\text { Prospectiv } \\
\text { ely } \\
\text { collected, } \\
\text { multicente } \\
\text { r database } \\
(2013- \\
2017) \text { of } \\
13 \text { spine } \\
\text { surgery } \\
\text { centers } \\
\text { across the } \\
\text { USA }\end{array}$ & $\begin{array}{l}\text { Surgery for CD: } \\
\text { Cervical kyphosis } \\
\text { (major angle } \\
>10^{\circ} \text { ), cervical } \\
\text { scoliosis (major } \\
\text { angle }<10^{\circ} \text { ), } \\
\text { C7SVA }>40 \mathrm{~mm} \\
\text { or CBVA }>25^{\circ}\end{array}$ & $\begin{array}{l}121 \mathrm{pts} \\
(61.47 \pm 9.8 \mathrm{yrs}) \\
48 \mathrm{men}, 73 \\
\text { women }\end{array}$ & $\begin{array}{l}\text { mCD-FI. } \\
\text { NF = 47.9\%; } \\
\mathrm{F}=46.3 \% \\
\mathrm{SF}=5.8 \%\end{array}$ & $\begin{array}{l}\text { SF: } \uparrow \text { overall } \\
\text { comorbidity } \\
\text { burden, } \\
\text { depression, } \\
\text { pulmonary disease } \\
\text { than NF. } \\
\text { F: } \uparrow \text { vascular } \\
\text { complication, } \\
\text { superficial surgical } \\
\text { site infection, } \\
\text { deterioration } \\
\text { patient-reported } \\
\text { measures of neck } \\
\text { pain, neck } \\
\text { disability, and } \\
\text { overall HRQoL, } \\
\text { LOS than NF. } \\
\text { SF: } \uparrow \text { cardiac } \\
\text { arrest, mortality, } \\
\text { deterioration in } \\
\text { patient-reported } \\
\text { measures of neck } \\
\text { pain, neck } \\
\text { disability, and } \\
\text { overall HRQoL, } \\
\text { LOS than F and } \\
\text { NF }\end{array}$ & $\begin{array}{l}\text { LOS; Complications; } \\
\text { HRQoL scores }\end{array}$ & 44 \\
\hline $\begin{array}{l}\text { Evaluation of } \\
\text { CD-FI to } \\
\text { predict p.o. } \\
\text { complications }\end{array}$ & $\begin{array}{l}\text { A } \\
\text { prospectiv } \\
\text { e, } \\
\text { multicente } \\
\text { r database } \\
(2013- \\
2018) \text { of } \\
13 \text { spine } \\
\text { surgery } \\
\text { centers } \\
\text { across } \\
\text { USA }\end{array}$ & $\begin{array}{l}\text { Surgery for CD: } \\
\text { cervical kyphosis } \\
\text { (major angle }> \\
10^{\circ} \text { ), C7SVA }>40 \\
\text { mm, TS-CL }>10^{\circ} \\
\text { or CBVA }>25^{\circ} ; \\
\text { minimum } 1-y r \\
\text { follow-up }\end{array}$ & $\begin{array}{l}138 \text { pts }(61.0 \\
\text { yrs }) . \\
53 \text { men, } 85 \\
\text { women }\end{array}$ & CD-FI & $\begin{array}{l}\mathrm{F}: \uparrow \text { minor and } \\
\text { major } \\
\text { complications than } \\
\mathrm{NF}\end{array}$ & $\begin{array}{l}\text { Perioperative } \\
\text { complications }\end{array}$ & 45 \\
\hline
\end{tabular}




\begin{tabular}{|c|c|c|c|c|c|c|c|}
\hline $\begin{array}{l}\text { Evaluation of } \\
\text { ASA, mCCI } \\
\text { and mFI to } \\
\text { predict p.o. } \\
\text { complications }\end{array}$ & $\begin{array}{l}\text { ACS- } \\
\text { NSQIP } \\
\text { database } \\
(2011- \\
2014)\end{array}$ & $\begin{array}{l}\text { Surgery for PLF or } \\
\text { PLIF }\end{array}$ & $\begin{array}{l}16,495 \text { pts } \\
(60 \pm 13.5 \text { yrs }) . \\
7357 \text { men, } 9138 \\
\text { women }\end{array}$ & $\begin{array}{l}\mathrm{mFI} \\
\mathrm{NF}=39.2 \% \\
\mathrm{PF}=58.9 \% \\
\mathrm{~F}=1.9 \%\end{array}$ & $\begin{array}{l}\text { mFI and ASA: } \uparrow \\
\text { discriminative } \\
\text { ability of any, } \\
\text { severe and minor } \\
\text { complications, } \\
\text { LOS, infectious } \\
\text { complications, } \\
\text { discharge to } \\
\text { higher-level care } \\
\text { than mCCI. } \\
\text { ASA: The most } \\
\text { predictive } \\
\text { comorbidity index }\end{array}$ & $\begin{array}{l}\text { Severe complications } \\
\text { (coma, cardiac arrest, } \\
\text { death, DVT, MI, } \\
\text { postoperative } \\
\text { intubation, PE, return } \\
\text { to the operating room, } \\
\text { sepsis, stroke); Minor } \\
\text { complications (acute } \\
\text { kidney injury, anemia } \\
\text { requiring transfusion, } \\
\text { pneumonia, surgical } \\
\text { site infection, UTI, } \\
\text { wound dehiscence); } \\
\text { Any complications } \\
\text { (major or minor AEs); } \\
\text { Infectious } \\
\text { complications } \\
\text { (pneumonia, sepsis, } \\
\text { surgical site infection, } \\
\text { UTI, wound } \\
\text { dehiscence); LOS; } \\
\text { Discharge to higher } \\
\text { level of care }\end{array}$ & 46 \\
\hline $\begin{array}{l}\text { Evaluation of } \\
\text { mFI to predict } \\
\text { mortality, } \\
\text { serious and } \\
\text { overall } \\
\text { complications }\end{array}$ & $\begin{array}{l}\text { ACS- } \\
\text { NSQIP } \\
\text { database } \\
(2010- \\
2014)\end{array}$ & Surgery for ALIF & $\begin{array}{l}3920 \text { pts (not } \\
\text { reported). } \\
\text { Not reported }\end{array}$ & $\begin{array}{l}\mathrm{mFI} \\
\mathrm{NF}=51.66 \% \\
\mathrm{PF}=47.09 \% \\
\mathrm{~F}=1.25 \%\end{array}$ & $\begin{array}{l}\mathrm{F}: \uparrow \text { any } \\
\text { complications, } \\
\text { pulmonary } \\
\text { complications than } \\
\mathrm{NF}\end{array}$ & $\begin{array}{l}\text { Complications (death, } \\
\text { pulmonary, renal, } \\
\text { CNS, wound and } \\
\text { cardiac complications, } \\
\text { venous } \\
\text { thromboembolism, } \\
\text { UTI, sepsis, graft } \\
\text { failure, blood } \\
\text { transfusions); Return } \\
\text { to the OR; LOS }\end{array}$ & 47 \\
\hline $\begin{array}{l}\text { Evaluation of } \\
\text { mFI to predict } \\
\text { p.o. } \\
\text { complications, } \\
\text { mortality }\end{array}$ & $\begin{array}{l}\text { ACS- } \\
\text { NSQIP } \\
\text { database } \\
(2005- \\
2012)\end{array}$ & $\begin{array}{l}\text { Surgery for lumbar } \\
\text { spinal fusion } \\
\text { procedures (PLF, } \\
\text { PLIF, TLF, TLIF) }\end{array}$ & $\begin{array}{l}6094 \text { pts } \\
(60 \pm 13.9 \text { yrs }) . \\
2742 \text { men, } 3352 \\
\text { women }\end{array}$ & $\begin{array}{l}\mathrm{mFI} \\
\mathrm{NF}=37.18 \% \\
\mathrm{PF}=56.71 \% \\
\mathrm{SF}=6.10 \%\end{array}$ & $\begin{array}{l}\text { F: } \uparrow \text { mortality, } \\
\text { reoperation, LOS, } \\
\text { unplanned } \\
\text { readmission, } \\
\text { several p.o. } \\
\text { complications, } \\
\text { pulmonary, renal, } \\
\text { PE/DVT, sepsis, } \\
\text { UTI, blood } \\
\text { transfusion, } \\
\text { wound } \\
\text { complications }\end{array}$ & $\begin{array}{l}\text { P.o. complications } \\
\text { (pneumonia, sepsis, } \\
\text { DVT, PE, wound } \\
\text { complication, deep } \\
\text { infection, CNS } \\
\text { complication, } \\
\text { sepsis/septic shock, } \\
\text { cardiac arrest, acute } \\
\text { renal failure, UTI); } \\
\text { Mortality occurring } \\
\text { within } 30 \text { days; } \\
\text { Reoperation; } \\
\text { Unplanned } \\
\text { reoperation; } \\
\text { Readmission; LOS }\end{array}$ & 48 \\
\hline $\begin{array}{l}\text { Evaluation of } \\
\text { mFI-5 to } \\
\text { predict 30-day } \\
\text { p.o. surgical } \\
\text { and medical } \\
\text { complications, } \\
\text { readmissions, } \\
\text { non-home } \\
\text { discharge and } \\
\text { mortality }\end{array}$ & $\begin{array}{l}\text { ACS- } \\
\text { NSQIP } \\
\text { database } \\
(2012- \\
2016)\end{array}$ & $\begin{array}{l}\text { Surgery for } \\
\text { elective PLFs for } \\
\text { lumbar spinal } \\
\text { stenosis, } \\
\text { spondylolisthesis, } \\
\text { degenerative disc } \\
\text { disease, } \\
\text { spondylosis }\end{array}$ & $\begin{array}{l}23,516 \text { pts }(\geq \\
18 \text { yrs }) . \\
10764 \text { men, } \\
12752 \text { women }\end{array}$ & $\begin{array}{l}\mathrm{mFI}-5 \\
\mathrm{NF}=38.11 \% \\
\mathrm{PF}=42.80 \% \\
\mathrm{~F}=19.10 \%\end{array}$ & $\begin{array}{l}\mathrm{PF}=\uparrow \text { any } \\
\text { complications, } \\
\text { medical } \\
\text { complications, 30- } \\
\text { day readmissions, } \\
\text { non-home } \\
\text { discharge than NF. } \\
\mathrm{F}=\uparrow \text { any } \\
\text { complications, } \\
\text { superficial and } \\
\text { deep SSI, } \\
\text { unplanned } \\
\text { reoperation, } \\
\text { medical } \\
\text { complications } \\
\text { (pneumonia, } \\
\text { unplanned } \\
\text { intubation, } \\
\text { postoperative vent } \\
\text { use, progressive } \\
\text { renal } \\
\text { insufficiency, } \\
\text { acute renal failure, }\end{array}$ & $\begin{array}{l}\text { Any complications; } \\
\text { Superficial SSI; Deep } \\
\text { SSI; Organ/space SSI; } \\
\text { Wound dehiscence; } \\
\text { Unplanned } \\
\text { reoperations; Medical } \\
\text { complications } \\
\text { (pneumonia, } \\
\text { unplanned intubation, } \\
\text { postoperative } \\
\text { ventilator use, } \\
\text { progressive renal } \\
\text { insufficiency, acute } \\
\text { renal failure, UTI, } \\
\text { stroke, MI, bleeding } \\
\text { requiring transfusion, } \\
\text { sepsis and septic } \\
\text { shock); 30-day } \\
\text { readmissions; } \\
\text { Mortality; Non-home } \\
\text { discharge }\end{array}$ & 49 \\
\hline
\end{tabular}




\begin{tabular}{|c|c|c|c|c|c|c|c|}
\hline & & & & & $\begin{array}{l}\text { UTI, CVA/stroke, } \\
\text { MI, bleeding } \\
\text { transfusions, } \\
\text { sepsis, septic } \\
\text { shock), 30-day } \\
\text { readmissions, non- } \\
\text { home discharge } \\
\text { than NF and PF }\end{array}$ & & \\
\hline $\begin{array}{l}\text { Evaluation of } \\
\text { frailty in LSS }\end{array}$ & $\begin{array}{l}\text { Spinal } \\
\text { center of a } \\
\text { tertiary- } \\
\text { care } \\
\text { teaching } \\
\text { hospital } \\
\text { database } \\
(2014- \\
2017)\end{array}$ & $\begin{array}{l}\text { LSS with a stenotic } \\
\text { lesion in the } \\
\text { lumbar spine }\end{array}$ & $\begin{array}{l}142 \mathrm{pts} \\
(72.1 \pm 6.9 \mathrm{yrs}) \\
42 \text { men, } 100 \\
\text { women }\end{array}$ & $\begin{array}{l}\text { Fried criteria } \\
N F=11.97 \% \\
P F=46.48 \% \\
F=41.55 \%\end{array}$ & $\begin{array}{l}\mathrm{F}: \uparrow \text { disability; } \downarrow \\
\text { quality of life than } \\
\mathrm{R} \text { and PF }\end{array}$ & ODI, EQ-5D & 50 \\
\hline $\begin{array}{l}\text { Evaluation of } \\
\text { frailty to } \\
\text { predict } \\
\text { perioperative } \\
\text { morbidity and } \\
\text { mortality }\end{array}$ & $\begin{array}{l}\text { ACS- } \\
\text { NSQIP } \\
\text { database } \\
(2007- \\
2012)\end{array}$ & $\begin{array}{l}\text { Surgery for spinal } \\
\text { decompression } \\
\text { with or without } \\
\text { fusion or VP/KP, } \\
\text { for thoracic } \\
\text { fractures with or } \\
\text { without SCI (T1- } \\
\text { T6, T7-T12), } \\
\text { lumbar fracture } \\
\text { with or without } \\
\text { cauda equine } \\
\text { injury }\end{array}$ & $\begin{array}{l}303 \text { pts } \\
(66.55 \pm 15.5 \\
\text { yrs }) . \\
138 \text { men, } 165 \\
\text { women }\end{array}$ & $\mathrm{mFI}$ & $\begin{array}{l}\mathrm{F}: \uparrow \\
\text { complications, 30- } \\
\text { day mortality than } \\
\mathrm{NF}\end{array}$ & $\begin{array}{l}\text { Perioperative } \\
\text { complication (30-day } \\
\text { mortality, } \\
\text { intraoperative events, } \\
\text { acute renal failure, } \\
\text { ventilator use for over } \\
48 \mathrm{~h} \text {, cerebrovascular } \\
\text { accident or stroke, MI, } \\
\text { cardiac arrest, PE, } \\
\text { sepsis, septic shock, } \\
\text { coma for over } 24 \mathrm{~h} \text {, } \\
\text { unplanned re- } \\
\text { intubation); Operative } \\
\text { time }\end{array}$ & 51 \\
\hline $\begin{array}{l}\text { Evaluation of } \\
\text { mFI to predict } \\
\text { p.o. morbidity } \\
\text { and mortality }\end{array}$ & $\begin{array}{l}\text { ACS- } \\
\text { NSQIP } \\
\text { database } \\
(2006- \\
2010)\end{array}$ & $\begin{array}{l}\text { Lumbar } \\
\text { laminectomy and } \\
\text { discectomy, } \\
\text { lumbar fusion, } \\
\text { anterior cervical } \\
\text { decompression, } \\
\text { anterior cervical } \\
\text { fusion, cervical } \\
360^{\circ} \text { fusion, } \\
\text { cervical } \\
\text { laminectomy and } \\
\text { fusion, thoracic } \\
\text { decompression and } \\
\text { fusion }\end{array}$ & $\begin{array}{l}18294 \text { pts (not } \\
\text { specified). } \\
9513 \text { men, } 8781 \\
\text { women }\end{array}$ & $\mathrm{mFI}$ & $\begin{array}{l}\mathrm{F}: \uparrow \text { at least } 1 \\
\text { infection, } \\
\text { mortality, surgical } \\
\text { site infections, } \\
\text { Clavien IV } \\
\text { complications than } \\
\mathrm{NF}\end{array}$ & $\begin{array}{l}\text { P.o. complications } \\
\text { (wound infection, any } \\
\text { infection, Clavien IV } \\
\text { complications); 30-day } \\
\text { mortality }\end{array}$ & 52 \\
\hline $\begin{array}{l}\text { Evaluation of } \\
\text { mFI-5 to } \\
\text { predict } \\
\text { comorbidities } \\
\text { and p.o. } \\
\text { complications }\end{array}$ & $\begin{array}{l}\text { ACS- } \\
\text { NSQIP } \\
\text { database } \\
(2006- \\
2015)\end{array}$ & $\begin{array}{l}\text { Kyphoplasty for } \\
\text { vertebral, lumbar } \\
\text { or thoracic } \\
\text { augmentation, } \\
\text { percutaneous } \\
\text { vertebral or lumbar } \\
\text { augmentation }\end{array}$ & $\begin{array}{l}2465 \text { pts }(74 \\
\text { yrs). } \\
735 \text { men, } 1730 \\
\text { women }\end{array}$ & $\begin{array}{l}\mathrm{mFI}-5 \\
\mathrm{NF}=26 \% \\
\mathrm{PF}=46.6 \% \\
\mathrm{~F}=29.4 \%\end{array}$ & $\begin{array}{l}\mathrm{F}: \uparrow \text { at least } 1 \\
\text { complication, } \\
\text { readmission rate, } \\
\text { LOS, discharged } \\
\text { to a location other } \\
\text { than home than } \\
\text { NF }\end{array}$ & $\begin{array}{l}\text { Complications } \\
\text { (cardiac, pulmonary, } \\
\text { wound, infection, } \\
\text { hematology, renal); } \\
\text { Other complications } \\
\text { (Stroke/cerebrovascula } \\
\text { r incident, need for } \\
\text { ventilator }>48 \text { hours, } \\
\text { septic shock, sepsis, } \\
\text { UTI); 30-day } \\
\text { readmission; 30-day } \\
\text { reoperation; LOS; } \\
\text { Adverse hospital } \\
\text { discharge }\end{array}$ & 53 \\
\hline $\begin{array}{l}\text { Evaluation of } \\
\text { frailty to } \\
\text { predict p.o. } \\
\text { functional } \\
\text { recovery and } \\
\text { cognition }\end{array}$ & $\begin{array}{l}\text { Mount } \\
\text { Sinai } \\
\text { Electronic } \\
\text { Schedulin } \\
\text { g system } \\
(2013- \\
2014)\end{array}$ & $\begin{array}{l}\text { Surgery at cervical } \\
\text { and lumbar levels; } \\
\text { ASA status I-III }\end{array}$ & $\begin{array}{l}100 \text { pts }(71 \\
\text { yrs). } \\
63 \text { men, } 37 \\
\text { women }\end{array}$ & $\begin{array}{l}\text { FRAIL scale } \\
\mathrm{NF}=26 \% \\
\mathrm{PF}=56 \% \\
\mathrm{~F}=18 \%\end{array}$ & $\begin{array}{l}\mathrm{R} \text { and PF: } \uparrow \\
\text { cognitive recovery } \\
\text { at } 3 \text { mo after } \\
\text { surgery than F. } \\
\mathrm{PF}: \downarrow \text { functional } \\
\text { recovery than } \mathrm{F} \\
\text { and } \mathrm{R} \text { at } 3 \text { mo }\end{array}$ & $\begin{array}{l}\text { Cognitive recovery; } \\
\text { ADL }\end{array}$ & 54 \\
\hline $\begin{array}{l}\text { Evaluation of } \\
\text { FP and FI to } \\
\text { predict p.o. } \\
\text { complications, } \\
\text { LOS, }\end{array}$ & $\begin{array}{l}\text { SAGES } \\
\text { prospectiv } \\
\text { e cohort } \\
\text { study }\end{array}$ & $\begin{array}{l}\text { Elective surgery: } \\
\text { lumbar, cervical or } \\
\text { sacral laminectomy }\end{array}$ & $\begin{array}{l}122 \mathrm{pts} \\
(76.8 \pm 5.2 \mathrm{yrs}) \\
165 \mathrm{men}, 250 \\
\text { women }\end{array}$ & $\begin{array}{l}\mathrm{FI} \\
\mathrm{NF}=21 \% \\
\mathrm{PF}=38 \% \\
\mathrm{~F}=41 \% \\
\mathrm{FP}\end{array}$ & $\begin{array}{l}\text { Moderate } \\
\text { concordance } \\
\text { between FP and } \\
\text { FI. }\end{array}$ & $\begin{array}{l}\text { P.o. medical and } \\
\text { surgical complications; } \\
\text { LOS; Discharge to } \\
\text { PAC; Readmission }\end{array}$ & 55 \\
\hline
\end{tabular}




\section{discharge to (2010- \\ PAC and 30- $\quad$ 2013) \\ day hospital}

readmission

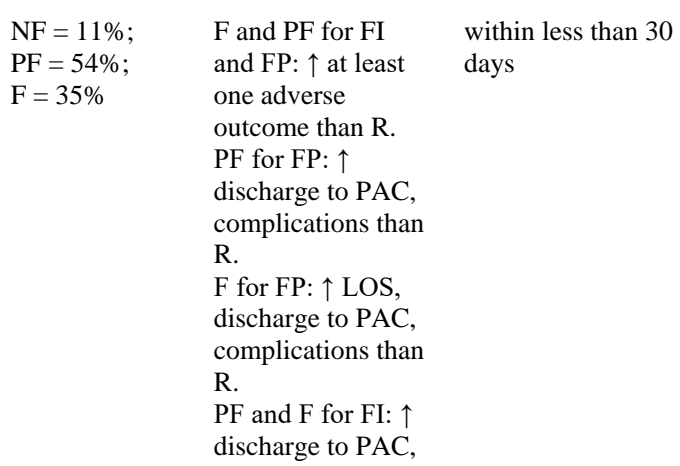

F and PF for FI and FP: $\uparrow$ at least one adverse outcome than $\mathrm{R}$. PF for FP: $\uparrow$ discharge to PAC, complications than $\mathrm{R}$.

F for FP: $\uparrow$ LOS, discharge to PAC, complications than $\mathrm{R}$.

$\mathrm{PF}$ and $\mathrm{F}$ for FI: $\uparrow$ discharge to PAC,

within less than 30

days LOS than $\mathrm{R}$

$\mathrm{F}: \uparrow \mathrm{ODI} ; \downarrow \mathrm{EQ}-$

and PF.

PF: $\uparrow$ ODI; $\downarrow$ EQ-

5D-5L than NF.

$\geq 3$ vertebral

fractures $=\uparrow \mathrm{F}$
5D-5L than NF
ODI; EQ-5D-5L

56

2017)

\begin{abstract}
ACS-NSQIP = American College of Surgeons National Surgical Quality Improvement Program; ADL = activities of daily living; AEs = adverse events; ALIF = anterior lumbar interbody fusion; ASA = American Society of Anesthesiologists; ASD = Adult Spinal Deformity; ASD-FI = Adult Spinal Deformity Frailty Index; BMI = body mass index; C7SVA = C7 sagittal vertical axis; CBVA = chin-brow vertical angle; CD = cervical deformity; CD-FI = Cervical deformity frailty index; CNS = central nervous system; CVA = cerebrovascular accident; DSD = degenerative spine disease; DVT = deep vein thrombosis; EQ-5D = EuroQol 5-dimension; ESSG = European Spine Study Group; F = frailty; FI = frailty index; FP = frailty phenotype; f-up = follow-up; $\mathrm{G}=$ good frailty control; $\mathrm{HRQoL}=$ Health related quality of life; ISSG = International Spine Study Group; LOS $=$ length of stay; LSS = lumbar spinal stenosis; $\mathrm{mCCI}=$ modified Charlson Comorbidity Index; $\mathrm{mCD}-\mathrm{FI}$ = modified cervical deformity frailty index; $\mathrm{mFI}=$ modified Frailty Index; MI = myocardial infarction; MSTFI = Metastatic Spinal Tumor Frailty Index; NF = not frailty; NIS = Nationwide Inpatient Sample; ODI $=$ Oswestry Disability Index; OR $=$ operative room; OVCF $=$ osteoporotic vertebral compression fracture; p.o. $=$ postoperative; $\mathrm{PAC}=$ postacute institutional care; $\mathrm{PC}=$ poor frailty control; $\mathrm{PE}=$ pulmonary embolism; $\mathrm{PF}=$ pre-frailty; $\mathrm{PI}-\mathrm{LL}=$ pelvic incidencelumbar lordosis; $\mathrm{PJK}=$ proximal junctional kyphosis; $\mathrm{PLF}=$ posterior lumbar fusion; $\mathrm{PLIF}=$ posterior lumbar interbody fusion; $\mathrm{pts}=$ patients; $\mathrm{R}=$ robust; SAGES = Successful Aging after Elective Surgery; SCB = substantial clinical benefit; SCI = Spinal Cord Injury; SF = severe frailty; SF36 PCS = 36-Item Short Physical Component Summary; SRS22 = Schwab-Scoliosis Research Society; SSI = surgical site infection; STFI = Spinal tumor frailty index; TLF = transforaminal lumbar fusion; TLIF = transforaminal lumbar interbody fusion; TS-CL = thoracic slope-cervical lordosis; $\mathrm{UTI}=$ urinary tract infections; $\mathrm{VP} / \mathrm{KP}=$ vertebroplasty/kyphoplasty; yrs = years
\end{abstract}

\section{Assessment of Methodological Quality}

Risks of bias assessments for each study were indicated in Table 2. Most of the studies showed an overall risk of bias low or moderate ( $\mathrm{n}=23$ studies, $79,3 \%$ ). Only a fraction of the studies ( $\mathrm{n}=3$ studies $(10,3 \%)[29,30,52]$ had a high risk, due to the lack of information for at least one aspect of the study attrition item [29, 30] or in analysis items, showing no statistical analysis [52].

All studies showed a low outcome measurement item because all studies had well described outcome measurement with a clear definition of the outcome, valid and reliable outcome measurements and the same method and setting of outcome measurement for all study participants. In addition, for all studies the Confounding Measurement and Account item was always moderate because the observed effect of the prognostic factors on outcome may be distorted by another factor related to the outcome.

\section{Spine tumors}

Five studies regarded spine benign or malignant primary tumors or metastatic ones [28-32].

More precisely, patients underwent surgery for benign or malignant neoplasms in vertebral column, sacrum and coccyx [28], spinal metastasis of primary tumors located in breast [29], lungs [29-31], thyroid [29, 30], kidney [2931 ], prostate [29, 30], bladder [30] or cervix [30] and one study described both primary or metastatic tumors allocated in extradural, intradural extramedullary and intramedullary sites [32].

\section{Frailty assessment in spine tumor studies}

Three different frailty indices were used for the identification and evaluation of frailty in patients affected by primary or metastatic spine tumors (Table 3 ), all of them are based on the accumulation of deficit model suggested by Rockwood. 
Table 2. QUIPS tool for assessing risk of bias in the clinical studies.

\begin{tabular}{|c|c|c|c|c|c|c|}
\hline Ref & $\begin{array}{l}\text { Study } \\
\text { Participation }\end{array}$ & $\begin{array}{l}\text { Study } \\
\text { Attrition }\end{array}$ & $\begin{array}{l}\text { Prognostic Factor } \\
\text { Measurement }\end{array}$ & $\begin{array}{l}\text { Outcome } \\
\text { Measurement }\end{array}$ & $\begin{array}{l}\text { Confounding Measurement } \\
\text { and Account }\end{array}$ & Analysis \\
\hline 28 & Low & Moderate & Low & Low & Moderate & Low \\
\hline 29 & Moderate & High & Moderate & Low & Moderate & Low \\
\hline 30 & Low & High & Low & Low & Moderate & Moderate \\
\hline 31 & Low & Moderate & Low & Low & Moderate & Low \\
\hline 32 & Moderate & Moderate & Low & Low & Moderate & Low \\
\hline 33 & Low & Moderate & Low & Low & Moderate & Low \\
\hline 34 & Moderate & Moderate & Moderate & Low & Moderate & Low \\
\hline 35 & Moderate & Moderate & Moderate & Low & Moderate & Low \\
\hline 36 & Moderate & Moderate & Moderate & Low & Moderate & Low \\
\hline 37 & Low & Moderate & Moderate & Low & Moderate & Low \\
\hline 38 & Low & Low & Low & Low & Moderate & Low \\
\hline 39 & Low & Low & Low & Low & Moderate & Low \\
\hline 40 & Low & Low & Low & Low & Moderate & Low \\
\hline 41 & Low & Low & Low & Low & Moderate & Low \\
\hline 42 & Moderate & Moderate & Low & Low & Moderate & Low \\
\hline 43 & Moderate & Moderate & Moderate & Low & Moderate & Low \\
\hline 44 & Low & Moderate & Moderate & Low & Moderate & Low \\
\hline 45 & Low & Moderate & Moderate & Low & Moderate & Low \\
\hline 46 & Low & Moderate & Low & Low & Moderate & Low \\
\hline 47 & Low & Moderate & Low & Low & Moderate & Low \\
\hline 48 & Low & Moderate & Low & Low & Moderate & Low \\
\hline 49 & Low & Moderate & Moderate & Low & Moderate & Low \\
\hline 50 & Low & Moderate & Moderate & Low & Moderate & Low \\
\hline 51 & Moderate & Moderate & Moderate & Low & Moderate & Low \\
\hline 52 & Moderate & Moderate & Moderate & Low & Moderate & High \\
\hline 53 & Low & Low & Low & Low & Moderate & Low \\
\hline 54 & Low & Low & Low & Low & Moderate & Moderate \\
\hline 55 & Low & Low & Low & Low & Moderate & Low \\
\hline 56 & Low & Low & Low & Low & Moderate & Low \\
\hline
\end{tabular}

Spinal Tumor Frailty Index (STFI) [28] and Metastatic Spinal Tumor Frailty Index (MSTFI) [29, 31] were respectively used in benign or malignant primary spine tumors [28] and in spinal metastases with a primary tumor located in breast, lungs, thyroid, kidney or prostate $[29,31]$. Both indices grouped patients into 4 frailty categories: no frailty (0), mild frailty (1), moderate frailty (2) and severe frailty $(\geq 3)$.

Modified Frailty Index (mFI) was employed in patients affected by spinal metastasis with a primary tumors in prostate, unknown sites, breast, lung, bladder, kidney, cervix, thyroid $[30,31]$ or primary and metastatic spine tumors in extradural, intradural extramedullary and intramedullary locations [32]. The cut-off for not frailty is 0 , that for pre-frailty is $0-0.21$ and that for frailty is $\geq 0.27$.

\section{Results in spine tumor studies}

Mild, moderate and severe frailty significantly increased all complications and LOS in hospital in 1589 patients with age between 28 and 61 years [28]. Similarly, moderate and severe frailty significantly increased mortality, while mild, moderate and severe frailty were associated with major complications and LOS in 4583 patients (age 54-70 years) [29].

In 41 [30], 108 [31] and 2170 [32] patients, with a mean age of 60 years, one group of authors did not find correlation between frailty status and mortality after surgery [30], while other authors showed that frail patients had higher mortality and LOS than not frail ones [32]. MSTFI was also compared with $\mathrm{mFI}$, underling that $\mathrm{mFI}$ correlated with complications, while MSTFI with mortality [31].

\section{Other spine diseases}

Twenty-five clinical studies regarded patients who underwent surgery for ASD (7/25 studies) [33-39], DSD (3/25 studies) [40-42], CD (3/25 studies) [43-45], lumbar spine disease (5/25 studies) [46-50] and diseases involving different spine levels (5/25 studies) [51-55], 
that comprised also patients with osteoporotic vertebral

fractures (1/25 study) [56].

Table 3. Frailty indices employed in the 29 clinical studies included in the systematic review.

\begin{tabular}{|c|c|c|c|c|c|}
\hline & $\begin{array}{l}\text { Frailty Index } \\
\text { name }\end{array}$ & $\begin{array}{l}\text { Frailty index } \\
\text { acronym }\end{array}$ & Items & Frailty scale & Ref. \\
\hline \multirow[t]{5}{*}{$\begin{array}{l}\text { Accumulation } \\
\text { of deficit } \\
\text { model }\end{array}$} & $\begin{array}{l}\text { Spinal tumor } \\
\text { frailty index }\end{array}$ & STFI & $\begin{array}{l}\text { 1) Anemia; 2) congestive heart failure; 3) } \\
\text { chronic obstructive pulmonary disease; 4) } \\
\text { coagulopathy; 5) electrolyte abnormalities; 6) } \\
\text { pulmonary circulation disorders; 7) renal } \\
\text { failure; 8) malnutrition; 9) pathologic fractures }\end{array}$ & $\begin{array}{l}\text { NF: } 0 \\
\text { Mild Frailty: } \\
1 ; \\
\text { Moderate } \\
\text { frailty: } 2 \\
\text { SF: } \geq 3\end{array}$ & 28 \\
\hline & $\begin{array}{l}\text { Metastatic } \\
\text { Spinal Tumor } \\
\text { Frailty Index }\end{array}$ & MSTFI & $\begin{array}{l}\text { 1) Anemia; 2) Chronic lung disease; 3) } \\
\text { Coagulopathy; 4) Electrolyte abnormalities; 5) } \\
\text { Pulmonary circulation disorders; 6) Renal } \\
\text { failure; 7) Malnutrition; 8) Emergent/urgent } \\
\text { case; 9) Anterior or combined surgical } \\
\text { approach }\end{array}$ & $\begin{array}{l}\text { NF: } 0 \\
\text { Mild Frailty: } \\
1 ; \\
\text { Moderate } \\
\text { Frailty: } 2 \\
\text { SF }: \geq 3\end{array}$ & 29,31 \\
\hline & $\begin{array}{l}\text { Modified Frailty } \\
\text { Index }\end{array}$ & $\mathrm{mFI}$ & $\begin{array}{l}\text { 1) Non-independent functional status; } 2 \text { ) } \\
\text { history of diabetes mellitus; } 3 \text { ) history of } \\
\text { chronic obstructive pulmonary disease; } 4 \text { ) } \\
\text { history of congestive heart failure; 5) history of } \\
\text { myocardial infarction; 6) history of } \\
\text { percutaneous coronary intervention, cardiac } \\
\text { surgery, or angina; 7) hypertension requiring } \\
\text { the use of medication; 8) peripheral vascular } \\
\text { disease or rest pain; 9) impaired sensorium; 10) } \\
\text { transient ischemic attack or cerebrovascular } \\
\text { accident w/o residual deficit; } 11 \text { ) } \\
\text { cerebrovascular accident w/o deficit }\end{array}$ & $\begin{array}{l}\text { NF: } 0 \\
\text { PF: } 0-0.21 \\
F: \geq 0.27\end{array}$ & $\begin{array}{l}30,31, \\
32,37, \\
38-42, \\
46-48, \\
51,52\end{array}$ \\
\hline & $\begin{array}{l}\text { Adult spinal } \\
\text { deformity frailty } \\
\text { index }\end{array}$ & ASD-FI & $\begin{array}{l}\text { 1)Health deficits documented by physician: }>3 \\
\text { medical problems; BMI <18.5 or }>30 \mathrm{~kg} / \mathrm{m} 2 \text {; } \\
\text { Cancer; Cardiac disease; Currently on } \\
\text { disability; Depression; Diabetes; Hypertension; } \\
\text { Liver disease; Lung disease; Osteoporosis; } \\
\text { Peripheral vascular disease; Previous blood clot } \\
\text { (DVT/PE/stroke); Smoking status. } \\
\text { 2)Health deficits patient-reported: Bladder } \\
\text { incontinence; Bowel incontinence; } \\
\text { Deteriorating health this yr; Difficulty climbing } \\
1 \text { flight of stairs; Difficulty driving a car; } \\
\text { Difficulty getting dressed; Difficulty getting } \\
\text { in/out of bed; Difficulty sleeping >6 hrs; } \\
\text { Difficulty walking } 100 \text { yards; Difficulty w/o } \\
\text { light activity; Feeling downhearted/depressed } \\
\text { most of the time; Feeling tired most of the } \\
\text { time; Feeling worn out most of the time; } \\
\text { General health: fair/poor; Inability to bathe w/o } \\
\text { assistance; Inability to cheer up often; Inability } \\
\text { to do normal work/schoolwork/housework; } \\
\text { Inability to lift heavy objects; Inability to travel } \\
>1 \text { hr; Inability to walk w/o assistive device; } \\
\text { Leg weakness; Loss of balance; Not in } \\
\text { excellent health; Personal care dependency; } \\
\text { Restricted activity level; Restricted social life }\end{array}$ & $\begin{array}{l}\text { NF: }<0.3 \\
\text { F: } 0.3-0.5 \\
\text { SF: }>0.5\end{array}$ & $33-36$ \\
\hline & $\begin{array}{l}\text { Modified Frailty } \\
\text { Index } 5\end{array}$ & $\mathrm{mFI}-5$ & $\begin{array}{l}\text { 1) history of severe chronic obstructive } \\
\text { pulmonary disease; } 2 \text { ) congestive heart failure } \\
\text { within } 30 \text { days before surgery; } 3 \text { ) functional } \\
\text { health status prior to surgery (independent }\end{array}$ & $\begin{array}{l}\text { NF: } 0 \\
\text { PF: } 1 \\
F: \geq 2\end{array}$ & $\begin{array}{l}38,49, \\
53\end{array}$ \\
\hline
\end{tabular}




\begin{tabular}{|c|c|c|c|c|c|}
\hline & & & $\begin{array}{l}\text { versus partially or totally dependent); 4) } \\
\text { hypertension requiring medication; 5) diabetes } \\
\text { mellitus with oral agents or insulin }\end{array}$ & & \\
\hline & $\begin{array}{l}\text { Cervical } \\
\text { deformity frailty } \\
\text { index }\end{array}$ & CD-FI & $\begin{array}{l}\text { 1)Health deficits documented by physician: >3 } \\
\text { Medical problems; Anxiety; BMI <18.5 or } \\
\text { >30; Cancer; Cardiac disease; Cerebrovascular } \\
\text { disease; Currently receiving disability benefits; } \\
\text { Dementia; Depression; Diabetes; Liver disease; } \\
\text { Lung disease; Neuromuscular disease; } \\
\text { Osteoporosis; Pancreatic disease; Rheumatoid } \\
\text { arthritis; Smoker; Vascular disease; Venous } \\
\text { disease; Unsteady gait. } \\
\text { 2)Health deficits patient-reported: Bladder } \\
\text { incontinence; Bowel incontinence; Difficulty } \\
\text { driving; Difficulty getting dressed; Difficulty } \\
\text { reading; Difficulty sleeping >6 h; Difficulty } \\
\text { walking without assistive device; Feeling } \\
\text { anxious or depressed most of the time; Feeling } \\
\text { tired most of the time; Feeling weak most of } \\
\text { the time; Feeling worn out/exhausted most of } \\
\text { the time; General health <50; Inability to } \\
\text { concentrate; Inability to do normal } \\
\text { work/schoolwork/housework; Inability to } \\
\text { engage in normal recreational activity; Inability } \\
\text { to lift heavy objects; Inability to perform } \\
\text { normal activities; Inability to walk; Leg } \\
\text { weakness; Personal care dependency }\end{array}$ & $\begin{array}{l}\text { NF: }<0.2 \\
\text { F: } 0.2-0.4 \\
\text { SF: }>0.4\end{array}$ & 43,45 \\
\hline & $\begin{array}{l}\text { Modified } \\
\text { cervical } \\
\text { deformity frailty } \\
\text { index }\end{array}$ & mCD-FI & $\begin{array}{l}\text { 1)Lung disease; 2) BMI <18.5 kg/m2 or }>30 \\
\mathrm{~km} / \mathrm{m} 2 \text {; 3) Diabetes; 4) Depression; 5) Liver } \\
\text { disease; 6) Rheumatoid arthritis; 7) Venous } \\
\text { disease; 8) Unsteady gait; 9) Bladder } \\
\text { incontinence; 10) Leg weakness; 11) } \\
\text { Comorbidities; 12) Anxiety; 13) Bowel } \\
\text { incontinence; 14) Difficulty sleeping }>6 \mathrm{~h} ; 15 \text { ) } \\
\text { Inability to walk }\end{array}$ & $\begin{array}{l}\text { NF: }<0.3 \\
\text { F: } 0.3-0.5 \\
\text { SF: }>0.5\end{array}$ & 44 \\
\hline & Frailty Index & FI & $\begin{array}{l}\text { 1) Help Bathing; 2) Help Dressing; 3) Help } \\
\text { getting in/out of Chair; 4) Help Walking } \\
\text { around house; 5) Help Eating; 6) Help } \\
\text { Grooming; 7) Help Using Toilet; 8) Help } \\
\text { up/down Stairs; 9) Help lifting 10 lbs; 10) Help } \\
\text { Shopping; 11) Help with Housework; 12) Help } \\
\text { with meal Preparations; 13) Help taking } \\
\text { Medication; 14) Help with Finances; 15) Lost } \\
\text { more than 10 lbs in last year; 16) Self Rating of } \\
\text { Health; 17) How Health has changed in last } \\
\text { year; 18) Stayed in Bed at least half the day } \\
\text { due to health (in last month); 19) Cut down on } \\
\text { Usual Activity (in last month); 20) Walk } \\
\text { outside; 21) Feel Everything is an Effort; 22) } \\
\text { Feel Depressed; 23) Feel Happy; 24) Feel } \\
\text { Lonely; 25) Have Trouble getting going; 26) } \\
\text { High blood pressure; 27) Heart attack; 28) } \\
\text { CHF; 29) Stroke; 30) Cancer; 31) Diabetes; 32) } \\
\text { Arthritis; 33) Chronic Lung Disease; 34) } \\
\text { MMSE; 35) Peak Flow; 36) Shoulder Strength; } \\
\text { 37) BMI; 38) Grip Strength; 39) Usual Pace; } \\
\text { 40) Rapid Pace }\end{array}$ & $\begin{array}{l}\text { NF: } 0.15 \\
\text { PF: } 0.15- \\
0.24 \\
F: \geq 0.25\end{array}$ & 55 \\
\hline $\begin{array}{l}\text { Phenotypic } \\
\text { model }\end{array}$ & & FRAIL scale & $\begin{array}{l}\text { 1)fatigue over the past } 4 \text { months; } 2 \text { ) ability to } \\
\text { climb a flight of stairs unassisted; } 3 \text { ) ability to } \\
\text { walk two blocks unassisted; } 4 \text { ) medical } \\
\text { comorbidities; 5) loss of weight }\end{array}$ & $\begin{array}{l}\text { NF: } 0 \\
P F: 1-2 \\
F: 3-5\end{array}$ & 54 \\
\hline
\end{tabular}




\begin{tabular}{|c|c|c|c|c|}
\hline & $\begin{array}{l}\text { FRIED } \\
\text { criteria }\end{array}$ & $\begin{array}{l}\text { 1) weight loss; 2) exhaustion; 3) physical } \\
\text { inactivity; 4) slowness; 5) handgrip strength }\end{array}$ & $\begin{array}{l}\text { NF: } 0 \\
P F: 1-2 \\
F: \geq 3\end{array}$ & 50,56 \\
\hline $\begin{array}{l}\text { Frailty } \\
\text { Phenotype } \\
\text { criteria }\end{array}$ & FP criteria & $\begin{array}{l}\text { 1) Slow gait (3-m timed walk); 2) Weakness } \\
\text { (grip strength); 3) Low activity (energy } \\
\text { expenditure); 4) Involuntary weight loss; 5) } \\
\text { Exhaustion }\end{array}$ & $\begin{array}{l}\text { NF: } 0 \\
P F: 1-2 \\
F: \geq 3\end{array}$ & 55 \\
\hline
\end{tabular}

\section{Frailty assessment in spine disease studies}

As observed in Table 3, 9 frailty indices were employed to stratify patients affected by different spine diseases that needed surgery. mFI was yet employed in tumor section. Some of them are included in the accumulation of deficit model suggested by Rockwood (ASD-FI, mFI, mFI-5, CD-FI, mCD-FI), while the others follow the phenotypic model suggested by Fried (Fried criteria, FRAIL scale, and FP).

Adult Spinal Deformity Frailty Index (ASD-FI) [3336] stratified patients affected by ASD, into not frail (< $0.3)$, frail $(0.3-0.5)$ and severe frail $(>0.5)$ ones.

$\mathrm{mFI}$ and its trunked version, $\mathrm{mFI}-5$, were employed in patients affected by ASD [37-39], DSD [40-42], or subjected to posterior lumbar fusion (PLF) or posterior lumbar interbody fusion (PLIF) [46, 48], anterior lumbar interbody fusion (ALIF) [43, 47], thoracic fractures [51] or diseases at different spine levels [52], lumbar stenosis, spondylolisthesis, degenerative disc disease and spondylosis [49] and patients subjected to Kyphoplasty [53].

CD-FI [43, 45] divided patients, affected by CD, into not frail $(<0.2)$, frail $(0.2-0.4)$ and severe frail $(>0.4)$. Also, CD-FI had a trunked version, mCD-FI [44] with little differences, from CD-FI: not frail $(<0.3)$, frail $(0.3-$ $0.5)$ and severe frail $(>0.5)$.

Fried criteria [50, 56] and FRAIL scale [54] stratified patients, affected by stenotic lesions of the lumbar spine, vertebral fractures [56] and elective surgery at cervical and lumbar levels [54] into not frail (0), pre-frail (1-2) and frail ( $\geq 3)$ ones.

Finally, FI and FP were compared in one study [55], in patients that underwent to elective surgery at cervical and lumbar levels. FP divided patients into not frail (0), pre-frail (1-2) and frail $(\geq 3)$ ones, while FI into not frail (0.15), pre-frail $(0.15-0.24)$ and frail $(\geq 0.25)$ ones.

\section{Results in spine disease studies}

In 332 [33], 417 [34], 266 [35], 267 [36], 240 [37], 281 [38] and 1001 [39] patients of a mean age of 57 years and affected by ASD, frail and severe frail patients showed significantly higher intraoperative and postoperative complications, any complications, reoperation, proximal junctional kyphosis (PJK), wound dehiscence, deep wound infection, LOS and junctional kyphosis than not frail ones [34-36].

Frailty significantly increased mortality rate, blood transfusion, pulmonary embolism/deep vein thrombosis (PE/DVT), any postoperative complications and reoperation rate than not frailty [39]. It was also observed that frailty significantly increased the absolute changes in postoperative Oswestry Disability Index (ODI), 36-Item Short Physical Component Summary (SF36 PCS), leg pain and the proportion of patients that reached substantial clinical benefit for ODI, SF-36 PCS, leg pain score also in comparison to frailty and severe frailty [33].

Prefrail and frail patients in good control group or poorly controlled group experienced more perioperative complications and postoperative $\mathrm{C} 7$ sagittal vertical axis (C7SVA) than frail patients [37]. The control group of frailty was defined as treatment following the appropriate guidelines for each mFI factor [37]. Making a comparison between the classic $\mathrm{mFI}$ and the truncated form $\mathrm{mFI}-5$ items it was observed an excellent concordance, especially in the prediction of complications. The classic $\mathrm{mFI}$ was able to well correlate with total complications, perioperative complications, implant-related complications, while mFI-5 with severe complications [38].

In patients affected by DSD, frailty significantly increased risk of mortality, major complication, reoperation for postsurgical infection, LOS and discharge to a new facility $[40,41]$, even if one study did not find association with the incidence of postoperative complications [40]. A correlation between frailty and body mass index (BMI) in the prediction of postoperative complications was founded [42]. Underweight, obesity, prefrailty and frailty separately increased postoperative complications and underweight significantly increased prefrailty and frailty, while obesity only frailty. In addition, underweight and normal weight associated with pre frailty or frailty, overweight associated with frailty and obese associated with not frailty or frailty increased postoperative complications [42]. The number of patients were 12 [40], 52671 [41] and 1970 [42] with a mean age of 62 years.

An amount of 61 [43] and 121 [44] severe frail patients with a mean age of 61 years, with $C D$, showed higher major complication, medical complications, overall comorbidity burden, depression and pulmonary 
disease in comparison to not frail ones [43, 44] and cardiac arrest, mortality, deterioration in patient-reported measures of neck pain, neck disability and overall Health related quality of life (HRQoL) and LOS more than not frail and frail patients [44]. In addition, 121 [44] and 138 [45] frail patients with a mean age of 61 years significantly increased vascular complication, superficial surgical site infection, deterioration patient-reported measures of neck pain, neck disability, and overall HRQoL, LOS, minor and major complications than not frail ones [44, 45].

In lumbar spine diseases, frailty was significantly associated with increase in any complications, pulmonary complications, mortality, reoperation, LOS, unplanned readmission, several postoperative complications, PE/DVT, sepsis, urinary tract infections (UTI), blood transfusion and wound complications than not frailty [47, 48]. In comparison with American Society of Anesthesiologists (ASA) score, mFI was less predictive of postoperative comorbidities, even if both were associated with severe complications, LOS, infectious complications and discharge to higher-level care [46]. Frail patients increased any complications, disability, superficial and deep Surgical site infection (SSI), unplanned reoperation, medical complications (pneumonia, unplanned intubation, postoperative vent use, progressive renal insufficiency, acute renal failure, UTI, cerebrovascular accident (CVA)/stroke, myocardial infarction (MI), bleeding transfusions, sepsis, septic shock), 30-day readmissions, nonhome discharge, disability and poor HQoL more than not frail or pre-frail ones $[49,50]$.
The patients enrolled were 16495 [46], 3920 [47], 6094 [48], 23516 [49], and 142 [50] at a mean age of 64 years.

In 303 [51], 18294 [52] and 2465 [53] patients (mean age of 70 years), that underwent different spine level surgery, frailty significantly increased complication rate, 30-day mortality, at least 1 infection and surgical site infections [51, 52], at least 1 complication, readmission rate, LOS and discharged to a location other than home [53] than not frailty.

One hundred frail patients of 71 years showed significantly high reduction in cognitive recovery at 3 months after surgery than not frail and pre-frail ones, and pre-frail patients showed significant higher reduction in functional recovery 3 months after surgery not only in comparison to not frail, but also than frail patients [54].

Frailty index (FI) and frailty phenotype (FP) were compared, showing a moderate concordance because both indices observed that adverse outcomes significantly increased with frailty and pre-frailty, but FI was associated with increased discharge to postacute institutional care (PAC) and LOS in pre-frail and frail patients, while FP was associated with discharge to PAC and complications in pre-frailty and increased discharge to PAC, complications and LOS in frailty [55]. The patients were 122 with a mean age of 77 years.

Finally, frailty significantly increased ODI and decreased EuroQol 5-dimension questionnaire (EQ-5D5L) than not frailty and pre-frailty, calculated with Fried criteria in 59 patients with 73 years mean age [56].

Table 4. Studies addressing association between gender and frailty on spine pathologies.

\begin{tabular}{|c|c|c|c|c|}
\hline Frailty Index & Spine Disease & Outcome & Conclusions & Ref \\
\hline ASD-FI & ASD & $\begin{array}{l}\text { Female }=(88.1 \%) \mathrm{NF} ;(79.4 \%) \mathrm{F} ;(68.2 \%) \mathrm{SF} \\
p=0.028: \mathrm{NF} V s \text { F and SF; F Vs SF }\end{array}$ & $\begin{array}{l}\text { Frailty severity decreased } \\
\text { with female sex }\end{array}$ & 33 \\
\hline mCD-FI & $\mathrm{CD}$ & $\begin{array}{l}\text { Female }=(48.3 \%) \mathrm{NF} ;(71.4 \%) \mathrm{F} ;(71.4 \%) \mathrm{SF} \\
p=0.034: \mathrm{F} \text { and SF } V s \mathrm{NF}\end{array}$ & $\begin{array}{l}\text { Frailty severity increased } \\
\text { with female sex }\end{array}$ & 44 \\
\hline \multirow[t]{2}{*}{ mFI } & DSD & $\begin{array}{l}\text { Male }=(50.8 \%) \mathrm{NF} ;(53 \%) \mathrm{PF} ;(60.1 \%) \mathrm{F} \\
p<0.0005: \mathrm{F} V S \text { NF and PF. }\end{array}$ & $\begin{array}{l}\text { Frailty severity increased } \\
\text { with male sex }\end{array}$ & 41 \\
\hline & & $\begin{array}{l}\text { Male sex: } \uparrow \text { major complications, LOS, discharge } \\
\text { disposition than female sex } \\
p<0.0005 \text { Male } V S \text { female }\end{array}$ & & \\
\hline
\end{tabular}

\section{Gender and frailty}

Among the 29 studies, 3 addressed gender and its association with frailty or morbidity associated with some spine pathologies, such as ASD [33], DSD [41] and CD [44] (Table 4).

Regarding female gender, 2 studies evaluated frailty through ASD-FI [33] and mCD [44], showing contrasting results. More precisely, in 1 study the percentage of not frail women was significantly higher than that of frail and severe frail $(p=0.028)$ [33], while in the second study the opposite was observed: the percentage of not frail women was significantly lower than frail and severe frail ones [44]. In women affected by ASD, the severity of frailty decreased [33], while in those affected by $C D$, the severity of frailty increased [44]. 
As for male gender, in men affected by DSD, the severity of frailty, evaluated with $\mathrm{mFI}$, increased [41], because the percentage of frail men was significantly higher than not frail or pre frail ones $(p<0.0005)$. In addition, men showed higher major complications, LOS and discharge deposition than women $(\mathrm{p}<0.0005)$ [40].

\section{DISCUSSION}

The present systematic review summarizes the most frequent frailty indices used in literature to predict surgical outcomes in frail patients undergoing surgery for several different spine diseases: primary or metastatic tumors [28-32], ASD [33-39], DSD [40-42], CD [43-45], lumbar spine pathologies [46-50] or multilevel spine ones [51-56]. In this review, frailty indices are also correlated with the most common postoperative complications.

Frailty is defined as a reduction in physiological function, but it is separated from the concept of aging, leading to the conclusion that the physiological aging is distinct from the chronological one [57]. Frailty increases the health vulnerability and deterioration, especially in elderly and several different tolls are actually used to measure frailty.

Frailty prevalence increases with age and is correlated with disability, admission to hospital and mortality and it is observed an increase of its prevalence in patients undergoing surgery than the other patients $(42 \%-50 \% \mathrm{Vs}$ $4 \%-10 \%$ ) [58]. Frailty is a predictor of morbidity and mortality, more than age in elderly patients undergoing general surgery. Before surgery, the measurement of frailty and the stratification of patients become important for predicting complications, even if no consensus is found on which is the best frailty tool [59].

As the population ages, spine surgery needs to grow, to improve neurologic adverse events and pain. Since spine surgery is associated with complications or even mortality, it is important to select patients at higher risk before surgery, also with a view to reducing the costs of the health system $[60,61]$.

For these reasons, the present systematic review collects the most employed frailty indices able to evaluate the association of frailty and spine surgery outcomes for different spine diseases, to give an indication on which to use in the different cases before surgery.

Frailty indices are composed by items that comprise presence of some concomitant pathologies, the functional status, mood, cognitive capacity and health deficits measured by physician or by the patients. The cut-off that stratify the patients are obtained by dividing the number of the positive items for the total number of the items.

According to the results of this review, frailty indices based on accumulation of deficit model suggested by Rockwood (STFI, MSTFI, FI, mFI, ASD-FI, CD-FI,
$\mathrm{mFI}-5$ and mCD-FI, are ) were employed in most of the studies $[28-49,51-53,55]$ in comparison to the phenotypic model suggested by Fried (FRAIL scale, FRIED criteria and FP criteria) [50, 54-56].

In this review one group of spine pathologies, requiring surgery, is primary or metastatic tumors. The spinal metastasis incidence is between 30 and $70 \%$ among patients with primary tumors and $10 \%$ of the metastases undergoes surgery [62]. Three frailty indices are used: STFI [45] in primary tumors and MSTFI $[46,48]$ and $\mathrm{mFI}$ $[47,48,51]$ in metastatic ones. STFI and MSTFI are correlated with perioperative complications and MSTFI with mortality. Both indices are composed of 9 items that regard the presence of cardiovascular, respiratory, urinary and musculoskeletal system disorders and malnutrition. "Pathologic fracture" and "congestive heart failure" items of STFI are replaced by "emergent/urgent case" and "anterior or combined surgical approach" items in MSTFI.

$\mathrm{mFI}$ is the most famous and the most frequently used frailty index in literature also in other pathologies and it consists of 11 variables, that regard non-independent functional status and the history of concomitant pathologies. In spine tumors it is observed that frailty, measured with $\mathrm{mFI}$, is correlated with mortality and complications, even if only one study does not find a correlation between $\mathrm{mFI}$ and survival [30].

$\mathrm{mFI}$ and its truncated form $\mathrm{mFI}-5$, characterized by 5 items, are also in common in other spine pathologies that required surgery, identified in this review, including ASD [37-39], DSD [40-42] or patients undergoing PLF [46, 48, 49], PLIF [46, 48], ALIF [47], transforaminal lumbar fusion (TLF) [48], transforaminal lumbar interbody fusion (TLIF) [48], thoracic fractures [51], lumbar, cervical or thoracic procedures [52] and kyphoplasty [53].

ASD and other spine pathologies are usually associated with postoperative or perioperative complications, due to the invasiveness of surgical procedures, including large dissection, multilevel fusion or osteotomy [63].

In these cases, besides $\mathrm{mFI}$, other indices are used as ASD-FI, CD-FI, Fried criteria, FRAIL scale, FI and FP criteria.

ASD-FI, composed of 40 items, is divided into health deficits documented by physician (14 items) and health deficits patient-reported ones (26 items) and is employed in patients affected by ASD [33-36]. Similar frailty index is CD-FI, employed in patients suffered of CD [43, 45], composed by 40 items, health deficits documented by physician (20 items) and health deficits patient-reported ones (20 items). As mFI, also CD-FI possesses its truncated form, that comprises 15 items instead of 40 ones, that take into consideration the presence of diseases, 
BMI, weakness, anxiety and difficulty in sleeping or in walking [44].

FRIED criteria [50, 56] and FRAIL scale [54] are easier than the other ones because they are composed by 5 items and regard prevalently subjective functional performances: weight loss, exhaustion, physical inactivity, slowness and handgrip strength. The differences between the two indices is that FRAIL scale substitutes physical inactivity, slowness and handgrip strength with ability to climb a flight of stairs unassisted, ability to walk two blocks unassisted and medical comorbidities. FP, employed only by one study [55], takes into consideration similar items to FRIED and FRAIL indices. The same study that employed FP, compared it with FI (40 items) [55], that regards the need for help in carrying out daily actions, weight loss, mood and presence of pathologies.

All these frailty indices correlate well with perioperative or postoperative complications, mortality or overall survival, LOS, discharge to a facility that is not home. The most evaluated complications regard the respiratory (acute respiratory distress syndrome, pleurisy, pneumothorax, pulmonary collapse, reintubation, pneumonia, pulmonary embolism), cardiac (cardiac arrest, myocardial infarction, iatrogenic stroke) and urinary (acute renal failure) systems, sepsis, shock, intraoperative vascular, visceral or neurological injury, deep infection, prolonged intubation, return to the operating room, unplanned re-intubation, venous thromboembolism, coma, perioperative blood transfusion, urinary tract infection, wound dehiscence, pseudoarthrosis incidence, excessive bleeding and delirium.

One of the most awaited discussions in the scientific community concerns the method by which complications are collected. Unfortunately, many groups use the most disparate and personalized methods to collect complications, making a comparison difficult and often underestimating the real percentage of the problem. Chen et al. show that another fundamental point in collecting complications lies in the people who collect them. Surgeons underestimate the problem, while a group of external observers, not involved in surgery, seems the best people to collect complications in the right measure [64]. The two most frequent grading methods for complications in vertebral and orthopedic surgery are the Spine Adverse Event Severity System (SAVES) and Clavein-Dindo one. The first allows systematic prospective collection of postoperative adverse events in spinal surgery and is divided into 14 intraoperative and 22 postoperative events, the second is based on the therapy used to correct a specific complication and is divided into 7 grades [65, 66]. Only few studies employed these grading methods for complications, one study the SAVES [31], and 8 studies the Clavein-Dindo [37, 38, 41, 42, 49, 51-53].

The different definitions and classifications of complications by different investigators make difficult to compare studies, dividing complications into major (that lead to reoperation or permanent deficits) and minor ones. So, a limit of the present review could be the different methods used to record complications and the group that evaluates them. For this reason, frailty probably generates more complications than those published.

Most of the studies of the present review validate and evaluate one frailty index for each study. However, in 3 cases, the same study compared two frailty indices [31, $38,55]$. In patients with spinal metastases of primary tumors located in breast, lung and kidney, $\mathrm{mFI}$ and MSTFI were compared, showing that mFI correlated with postoperative complications, while MSTFI with mortality [31]. mFI and mFI-5 showed an excellent correlation across ASD surgery and were strong predictive for severe complications, but mFI correlated with total, perioperative and severe complications, while mFI-5 with severe complications [38]. Finally, a moderate accordance between FP and FI indices was observed. FP correlated with discharge to PAC and complications, while FI with discharge to PAC and LOS [55].

The patients included in the studies varied from a minimum of 41 [30] to a maximum of 52671 [41] and were both men and women, but a prevalence of studies enrolled more women than men [33-37, 39, 43-46, 48-51, $53,55,56]$. This seems to presage that, between the two genders, there is a prevalence of women who are frail compared to men. Still now, few studies identify gender differences in frailty. Three frailty indices are able to discriminate between males and females, but the results are discordant because frailty severity seems to decrease $[33,41]$ or increase [44] with female gender. More precisely, ASD-FI and $\mathrm{mFI}$ show that frailty severity decreases in women affected by ASD and DSD, respectively, and that men shows higher major complications, LOS and discharge disposition than women [33, 41]. Conversely, mCD-FI indicates that frailty severity increases more in women than in men affected by CD [44].

However, since only 3 studies dealt with gender difference, with heterogeneity in the study design, study participants, and spine pathologies, it was difficult to draw any significant conclusion regarding this theme.

Frailty is a prevalent age condition, but in this review $14 / 29$ studies considered also patients younger than 60 years [28, 29, 31, 32-39, 41, 42,49]. This reinforces the idea that physiological aging is distinct from the chronological one and that frailty indices can be applied at any age in pathologies of the spine. 


\section{Conclusion}

In summary, this systematic review identified 11 frailty indices that correlated well with complications of spine surgery outcomes, also with severe complications. Even if there is no consensus on which is best, $\mathrm{mFI}$ is the most employed and the most adaptable to all spine pathologies. Indeed, it is employed in metastatic tumors [30-32], ASD [37-39], DSD [40-42], lumbar pathologies [46-49] or multi-level pathologies [51-53]. In decreasing order of frequency the other indices are ASD-FI, exclusively in ASD pathology [33-36], CD-FI only in CD pathology [4345], MTSFI in metastatic tumors [29, 31], Fried criteria in lumbar spine pathologies [50] and vertebral fractures [56], FRAIL scale in cervical and lumbar pathologies [54], FP and FI in lumbar, cervical and sacral pathologies [55] and STFI in primary spine tumors [28].

Because it is one of the most complete indices, having 11 items that concern the functional status and the history of concomitant pathologies. At the same time, it is also less complex than other indices that may contain up to 40 items.

Clarity has not yet been made regarding the relationship between the frailty level and gender, even if a worsening of frailty is prevalently observed in women. Given the paucity of the studies regarding the comparison between different frailty indices in the same study and of the studies regarding the evaluation of gender in frailty, it will be mandatory to deepen these comparisons in future studies.

\section{Acknowledgements}

The work reported in this publication was granted by the Italian Ministry of Health, under the Aging Network of Italian Research Hospitals (IRCCS)".

\section{Competing interests}

The author declares no competing interests.

\section{References}

[1] Fried LP, Tangen CM, Walston J, A B Newman, C Hirsch, J Gottdiener, T Seeman, R Tracy, W J Kop, G Burke, M A McBurnie, Cardiovascular Health Study Collaborative Research Group (2001). Frailty in older adults: evidence for a phenotype. J Gerontol A Biol Sci Med Sci,56:M146-M156.

[2] Rockwood K, Bergman H (2021). FRAILTY: a report from the 3rd Joint Workshop of IAGG/WHO/SFGG, Athens, January 2012. Can Geriatr J, 15:31-6.

[3] Walston JD, Bandeen-Roche K (2015). Frailty: A tale of two concepts. BMC Medicine, 13:185.
Dent E, Kowal P, Hoogendijk EO (2016). Frailty measurement in research and clinical practice: A review. Europ J Int Med, 31:3-10.

[5] Buta BJ, Walston JD, Godino JG, Park M, Kalyani RR, Xue Q, Bandeen-Roche K, Varadhan R (2016). Frailty assessment instruments: Systematic characterization of the uses and contexts of highlycited instruments. Ageing Res Rev, 26:53-61.

[6] Collard RM, Boter H, Schoevers RA, Oude Voshaar RC (2012). Prevalence of frailty in communitydwelling older persons: a systematic review. J Am Geriatr Soc, 60:1487-1492.

[7] Syddall H, Roberts HC, Evandrou M, Cooper C, Bergman H, Sayer AA (2010). Prevalence and correlates of frailty among community-dwelling older men and women: findings from the Hertfordshire Cohort Study. Age Ageing, 39:197203.

[8] Siriwardhana DD, HardoonS, Rait G, Weerasinghe MC, Walter KR (2018). Prevalence of frailty and prefrailty among community-dwelling older adults in low-income and middle-income countries: a systematic review and meta-analysis. BMJ Open, 8:e018195.

[9] Hoogendijk EO, Afilalo J, Ensrud KE, Kowal P, Onder G, Fried LP (2019). Frailty: implications for clinical practice and public health. Lancet, 394:13651375.

[10] United Nations, Department of Economic and Social Affairs, Population Division. World population prospects 2019: highlights ST/ESA/SER.A/423, New York: United Nations (2019)

[11] Kalseth J, Halvorsen T (2020). Health and care service utilisation and cost over the life-span: a descriptive analysis of population data. BMC Health Serv Res, 20:435.

[12] Ensrud KE, Kats AM, Schousboe JT, Taylor BC, Vo TN, Cawthon PM, Hoffman R, Langsetmo L (2020). Osteoporotic Fractures in Men Study (MrOS). Frailty Phenotype and Healthcare Costs and Utilization in Older Men. J Am Geriatr Soc.

Robinson TN, Wu DS, Pointer L, Dunn CL, Cleveland JCJ, Moss M (2013). Simple frailty score predicts postoperative complications across surgical specialties. Am J Surg, 206:544-550.

[14] Mrdutt MM, Papaconstantinou HT, Robinson BD, Bird ET, Isbell CL (2019). Preoperative frailty and surgical outcomes across diverse surgical subspecialties in a large health care system. J Am Coll Surg, 228:482-490.

[15] Panayi AC, Orkaby AR, Sakthivel D, Endo Y, Varon D, Roh D, Orgil, DP, Neppl RL, Javedan H, Bhasin S, Sinha I (2019). Impact of frailty on outcomes in surgical patients: a systematic review and metaanalysis. Am J Surg, 218:393-400.

[16] Buigues C, Juarros-Folgado P, Fernández-Garrido J, Navarro-Martínez R, Cauli O (2015). Frailty syndrome and pre-operative risk evaluation: A systematic review. Arch Gerontol Geriatr, 61:309321. 
[17] Street JT, Lenehan BJ, DiPaola CP, Boyd MD, Kwon BK, Paquette SJ, Dvorak MF, Rampersaud YR, Fisher CG (2012). Morbidity and mortality of major adult spinal surgery. A prospective cohort analysis of 942 consecutive patients. Spine J, 12:22-34.

[18] Friedman GN, Benton JA, Echt M, De la Garza Ramos R, Shin JH, Coumans JVCE, Gitkind AI, Yassari R, Leveque JC, Sethi RK, Yanamadala V (2020). Multidisciplinary approaches to complication reduction in complex spine surgery: a systematic review. Spine J, 2020:S1529-9430(20)30143-1.

[19] Drazin D, Shirzadi A, Rosner J, Eboli P, Safee M, Baron EM, Liu JC, Acosta FL J (2011). Complications and outcomes after spinal deformity surgery in the elderly: review of the existing literature and future directions. Neurosurg Focus, 31:E3.

[20] Clarke MJ, Vrionis FD (2014). Spinal tumor surgery: management and the avoidance of complications. Cancer Control, 21:124-132.

[21] Eliyas JK, Karahalios D (2011). Surgery for degenerative lumbar spine disease. Dis Mon, 57:592606.

[22] Rubin DI (2007). Epidemiology and risk factors for spine pain. Neurol Clin, 25:353-371.

[23] National Spinal Cord Injury Statistical Center, Facts and Figures at a Glance. Birmingham, AL: University of Alabama at Birmingham, 2020

[24] Schousboe JT (2018). Vertebral Fracture Identification as Part of a Comprehensive Risk Assessment in Patients with Osteoporosis. Curr Osteoporos Rep, 16:573-583.

[25] Laufer I, Rubin DG, Lis E, Cox BW, Stubblefield MD, Yamada Y, Bilsky MH (2013). The NOMS framework: Approach to the treatment of spinal metastatic tumors. Oncologist, 18:744-51.

[26] Moskven E, Bourassa-Moreau E, Charest-Morin R, Flexman A, Street J (2018). The impact of frailty and sarcopenia on postoperative outcomes in adult spine surgery. A systematic review of the literature. Spine J, 18:2354-2369.

[27] Hayden JA, van der Windt DA, Cartwright JL, Cote P, Bombardier C (2013). Assessing Bias in Studies of Prognostic Factors. Ann Intern Med, 158:280-286.

[28] Ahmed AK, Goodwin CR, De la Garza-Ramos R, Kim RC, Abu-Bonsrah N, Xu R, Sciubba DM (2017). Predicting Short-Term Outcome After Surgery for Primary Spinal Tumors Based on Patient Frailty. World Neurosurg, 108:393-398.

[29] De la Garza Ramos R, Goodwin CR, Jain A, AbuBonsrah N, Fisher CG, Bettegowda C, Sciubba DM (2016). Development of a Metastatic Spinal Tumor Frailty Index (MSTFI) Using a Nationwide Database and Its Association with Inpatient Morbidity, Mortality, and Length of Stay After Spine Surgery. World Neurosurg, 95:548-555.

[30] Rothi IM, Deverall HH, Baker JF (2019). The modified Frailty Index does not correlate with survival in surgically treated patients with metastatic spine disease. J Clin Neurosci, 66:178-181.
[31] Bourassa-Moreau E, Versteeg A, Moskven E, Charest-Morin R, Flexman A, Ailon T, Dalkilic T, Fisher C, Dea N, Boyd M, Paquette S, Kwon B, Dvorak M, Street J (2020). Sarcopenia, but not frailty, predicts early mortality and adverse events after emergent surgery for metastatic disease of the spine. Spine J, 20:22-31.

[32] Lakomkin N, Zuckerman SL, Stannard B, Montejo J, Sussman ES, Virojanapa J, Kuzmik G, Goz V, Hadjipanayis CG, Cheng JS (2018). Preoperative Risk Stratification in Spine Tumor Surgery A Comparison of the Modified Charlson Index, Frailty Index, and ASA Score. Spine, 44:E782-E787.

[33] Reid DBC, Daniels AH, Ailon T, Miller E, Sciubba DM, Smith JS, Shaffrey CI, Schwab F, Burton D, Hart RA, Hostin R, Line B, Bess S, Ames CP (2018). Frailty and Health-Related Quality of Life Improvement Following Adult Spinal Deformity Surgery. World Neurosurg, 112:e548-e554.

[34] Miller EK, Neuman BJ, Jain A, Daniels AH, Ailon T, Sciubba DM, Kebaish KM, Lafage V, Scheer JK, Smith JS, Bess S, Shaffrey CI, Ames CP (2017). An assessment of frailty as a tool for risk stratification in adult spinal deformity surgery. Neurosurg Focus, 43:E3.

[35] Miller EK, Vila Casademunt A, Neuman BJ, Sciubba DM, Kebaish KM, Smith JS, Alanay A, Acaroglu ER, Kleinstück F, Obeid I, Sánchez Pérez Grueso FJ, Carreon LY, Schwab FJ, Bess S, Scheer JK, Lafage V, Shaffrey CI, Pellisé F, Ames CP (2018). External validation of the adult spinal deformity (ASD) frailty index (ASD FI). Europ Spine J, 27:2331-2338.

[36] Miller EK, Lenke LG, Neuman BJ, Sciubba DM, Kebaish KM, Smith JS, Qiu Y, Dahl BT, Pellisé F, Matsuyama Y, Carreon LY, Fehlings MG, Cheung KM, Lewis S, Dekutoski MB, Schwab FJ, BoachieAdjei O, Mehdian H, Bess S, Shaffrey CI, Ames CP (2018). External Validation of the Adult Spinal Deformity (ASD) Frailty Index (ASD-FI) in the Scoli-RISK-1 Patient Database. Spine, 43:14261431.

[37] Yagi M, Michikawa T, Hosogane N, Fujita N, Okada E, Suzuki S, Tsuji O, Nagoshi N, Asazuma T, Tsuji T, Nakamura M, Matsumoto M, Watanabe K (2018). Treatment for Frailty Does Not Improve Complication Rates in Corrective Surgery for Adult Spinal Deformity. Spine, 44:723-731.

[38] Yagi M, Michikawa T, Hosogane N, Fujita N, Okada E, Suzuki S, Tsuji O, Nagoshi N, Asazuma T, Tsuji T, Nakamura M, Matsumoto M, Watanabe K (2019). The 5-Item Modified Frailty Index Is Predictive of Severe Adverse Events in Patients Undergoing Surgery for Adult Spinal Deformity. Spine, 44:E1083-E1091.

[39] Leven DM, Lee NJ, Kothari P, Steinberger J, Guzman J, Skovrlj B, Shin JI, Caridi JM, Cho SK (2016). Frailty Index Is a Significant Predictor of Complications and Mortality After Surgery for Adult Spinal Deformity. Spine, 41:E1394-E1401. 
[40] Charest-Morin R, Street J, Zhang H, Rougheadd T, Ailon T, Boyd M, Dvorak M, Kwon B, Paquette S, Dea N, Fisher CG, Flexman AM (2018). Frailty and sarcopenia do not predict adverse events in an elderly population undergoing non-complex primary elective surgery for degenerative conditions of the lumbar spine. Spine J, 18:245-254.

[41] Flexman AM, Charest-Morin R, Stobart L, Street J, Ryerson CJ (2016). Frailty and postoperative outcomes in patients undergoing surgery for degenerative spine disease. Spine J, 16:1315-1323.

[42] Xu W, Zhang XM, Ke T, Cai HR, Gao X (2018). Modified Frailty Index and Body Mass Index as Predictors of Adverse Surgical Outcomes in Degenerative Spinal Disease. Turk Neurosurg, 28:897-903.

[43] Miller EK, Ailon T, Neuman BJ, Klineberg EO, Mundis Jr GM, Sciubba DM, Kebaish KM, Lafage V, Scheer JK, Smith JS, Hamilton DK, Bess S, Shaffrey CI, Ames CP (2018). Assment of a Novel Adult Cervical Deformity Frailty Index as a Component of Preoperative Risk Stratification. World Neurosurg, 109:e800-e806.

[44] Passias PG, Bortz CA, Segreto BA, Horn SR, Lafage R, Lafage V, Smith JS, Line B, Kim KJ, Eastlack R, Hamilton DK, Protopsaltis T, Hostin Jr RA, Klineberg EO, Burton DC, Hart RA, Schwab FJ, Bess S, Shaffrey CI, Ames CP (2018). Development of a Modified Cervical Deformity Frailty Index. A Streamlined Clinical Tool for Preoperative Risk Stratification. Spine, 44:169-176.

[45] Segreto FA, Passias PG, Brown AE, Horn SR, Bortz CA, Pierce KE, Alas H, Lafage V, Lafage R, Smith JS, Line BG, Diebo BG, Kelly MP, Mundis GM, Protopsaltis TS, Soroceanu A, Kim HJ, Klineberg EO, Burton DC, Hart RA, Schwab FJ, Bess S, Shaffrey CI, Ames CP (2019). The Influence of Surgical Intervention and Sagittal Alignment on Frailty in Adult Cervical Deformity. Oper Neurosurg, 18:583-589.

[46] Ondeck NT, Bohl DD, Bovonratwet P, McLynn RP, Cui JJ, Shultz BN, Lukasiewicz AM, Grauer JN (2018). Discriminative ability of commonly used indices to predict adverse outcomes after poster lumbar fusion: a comparison of demographics, ASA, the modified Charlson Comorbidity Index, and the modified Frailty Index. Spine J, 18:44-52.

[47] Phan K, Kim JS, Lee NJ, Somani S, Di Capua J, Kothari P, Leven D, Cho SK (2017). Frailty is associated with morbidity in adults undergoing elective anterior lumbar interbody fusion (ALIF) surgery. Spine J, 17:538-544.

[48] Leven DM, Lee NJ, Kim JS, Kothari P, Steinberger J, Guzman J, Skovrlj B, Shin JI, Phan K, Caridi JM, Cho SK (2017). Frailty Is Predictive of Adverse Postoperative Events in Patients Undergoing Lumbar Fusion. Global Spine J, 7:529-535.

[49] Weaver DJ, Malik AT, Jain N, Yu E, Kim J, Khan SN (2019). The Modified 5-Item Frailty Index: A Concise and Useful Tool for Assessing the Impact of
Frailty on Postoperative Morbidity Following Elective Posterior Lumbar Fusions. World Neurosurg, 124:e626-e632.

[50] Kim HJ, Park S, Park SH, Lee JH, Chang BS, Lee CK, Yeom JS (2019). The prevalence and impact of frailty in patients with symptomatic lumbar spinal stenosis. Europ Spine J, 28:46-54.

Kessler RA, De la Garza Ramos R, Purvis TE, Ahmed AK, Goodwin CR, Sciubba DM, Abd-El-Barr MM (2018). Impact of frailty on complications in patients with thoracic and thoracolumbar spinal fracture. Clin Neurol Neurosurg, 169:161-165.

[52] Ali R, Schwalb JM, Nerenz DR, Antoine HJ, Rubinfeld I (2016). Use of the modified frailty index to predict 30-day morbidity and mortality from spine surgery. J Neurosurg Spine, 25:537-541.

[53] Segal DN, Wilson JM, Staley C, Michael KW (2018). The 5-Item Modified Frailty Index Is Predictive of 30-Day Postoperative Complications in Patients Undergoing Kyphoplasty Vertebral Augmentation. World Neurosurg, 116:e225-e231.

[54] Rothrock RJ, Steinberger JM, Badgery H, Hecht AC, Cho SK, Caridi JM, Deiner S (2019). Frailty status as a predictor of 3-month cognitive and functional recovery following spinal surgery: a prospective pilot study. Spine J, 19:104-112.

[55] Cooper Z, Rogers SO, Ngo L, Guess J, Schmitt E, Jones RN, Ayres DK, Walston JD, Gill TM, Gleason LJ, Inouye SK, Marcantonio ER (2016). Comparison of Frailty Measures as Predictors of Outcomes After Orthopedic Surgery. Am Geriatr Soc, 64:2464-2471. Kim HJ, Park S, Park SH, Park J, Chang BS, Lee CK, Yeom JS (2018). Prevalence of Frailty in Patients with Osteoporotic Vertebral Compression Fracture and Its Association with Numbers of Fractures. Yonsei Med J, 59:317-324.

[57] Joseph B, Phelan H, Hassan A, Orouji Jokar T, O'Keeffe T, Azim A, Gries L, Kulvatunyou N, Latifi $\mathrm{R}$, Rhee $\mathrm{P}$ (2016). The impact of frailty on failure-torescue in geriatric trauma patients: a prospective study. J Trauma Acute Care Surg, 81:1150-1155.

[58] Iqbal J, Denvir M, Gunn J (2014). Frailty assessment in elderly people. Lancet, 381:1985-6.

[59] Kim SW, Han HS, Jung HW, Kim KI, Hwang DW, Kang SB, Kim CH (2014). Multidimensional frailty score for the prediction of postoperative mortality risk. JAMA Surg, 149:633-640.

[60] Ghori AK, Leonard DA, Schoenfeld AJ, Saadat E, Scott N, Ferrone ML, Pearson AM, Harris MB (2015). Modeling 1-year survival after surgery on the metastatic spine. Spine J, 15:2345-2350.

[61] Barbanti Bròdano G, Giavaresi G, Lolli F, Salamanna F, Parrilli A, Martini L, Griffoni C, Greggi T, Arcangeli E, Pressato D, Boriani S, Fini M (2014). Hydroxyapatite-Based Biomaterials Versus Autologous Bone Graft in Spinal Fusion: An In Vivo Animal Study. Spine,39: E661-E668.

[62] Park JH, Jeon SR (2013). Pre- and postoperative lower extremity motor power and ambulatory status 
of patients with spinal cord compression due to a metastatic spinal tumour. SPINE, 38:E798-802.

[63] Yagi M, Ohne H, Kaneko S, Machida M, Yato Y, Asazuma T (2018). Does corrective spine surgery improve the standing balance in patients with adult spinal deformity?. Spine J, 18:36-43.

[64] Chen BP, Garland K, Roffey DM, Poitras S, Dervin G, Lapner P, Phan P, Wai EK, Kingwell SP, Beaule PE (2017). Can Surgeons Adequately Capture Adverse Events Using the Spinal Adverse Events Severity System (SAVES) and OrthoSAVES?. Clin Orthop Relat Res, 475:253-260.

[65] Barbanti-Brodano G, Grifoni C, Halme J, Tedesco G, Terzi S, Bandiera S, Ghermandi R, Evangelisti G, Girolami M, Pipola V, Gasbarrini A, Falavigna A (2020). Spinal surgery complications: an unsolved problem-Is the World Health Organization Safety Surgical Checklist an useful tool to reduce them?. Eur Spine J, 29:927-936.

[66] Millstone DB, Perruccio AV, Badley EM, Rampersaud YR (2017). Factors Associated with Adverse Events in Inpatient Elective Spine, Knee, and Hip Orthopaedic Surgery. J Bone Joint Surg Am, 99:1365-1372. 